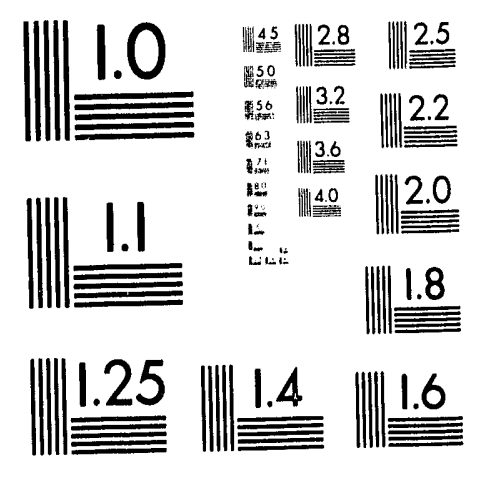



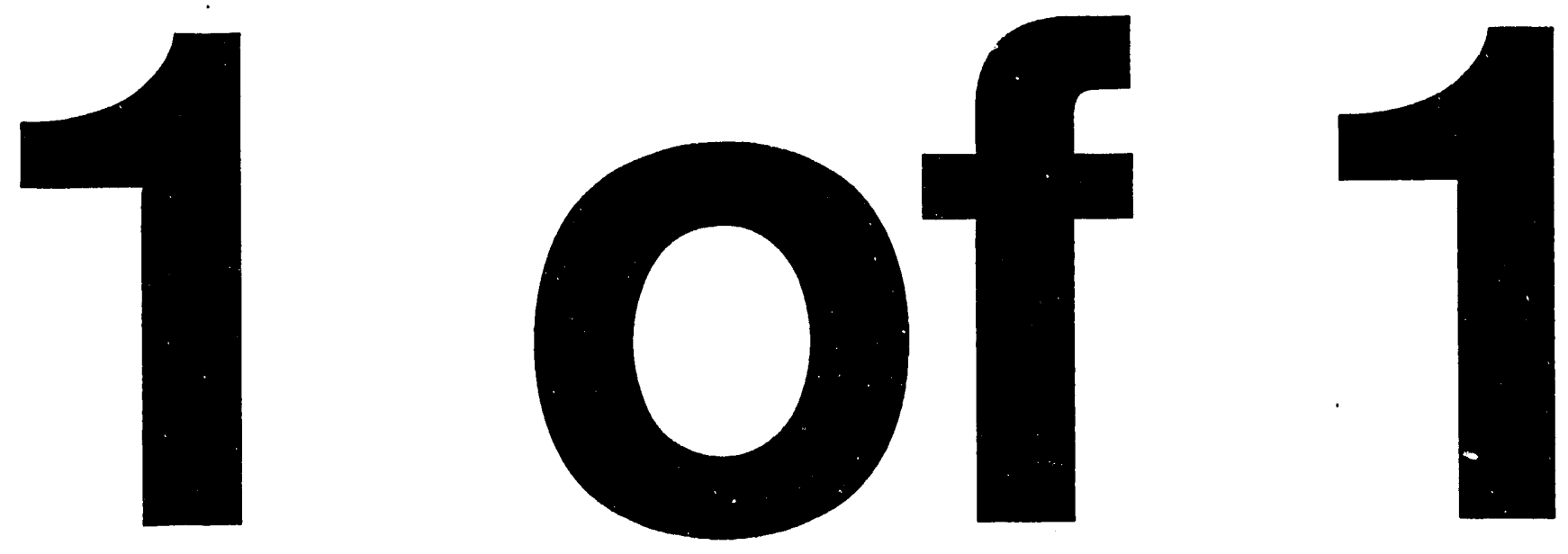


\title{
Radiation Environment and Shielding for the GEM Experiment at the SSC
}

M. Diwan, Y. Fisyak, N. Mokhov, D. Lee, L. Waters, W. Wilson

Y. Efremenko, B. Moore, M. Marx, C. Wuest, J. Rutherfoord, and V. Morgunov

\begin{abstract}
We have performed a comprehensive study of the radiation environment for the proposed GEM detector at the SSC. As a result of this study, we have developed a shielding scenario that will ensure that the detector will operate with its design performance for at least 10 years at the luminosity of $10^{33} \mathrm{~cm}^{-2} \mathrm{~s}^{-1}$.
\end{abstract}

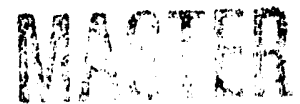




\subsection{INTRODUCTION}

As an integral part of the detector design we have performed simulation studies of the neutron, photon, and charged particle fluxes in GEM, and of the resultant doses and radioactivation of all major subsystems and neighboring accelerator components. As a result of these studies, we have developed a shielding and collimator configuration, and an overall layout of the experiment that will ensure that the detector will operate with its design performance for at least 10 years at the standard luminosity of $10^{33} \mathrm{~cm}^{-2} \mathrm{~s}^{-1}$. We are confident that modest design changes, resulting from continuing studies, will enable the detector (with the exception of the inner silicon tracker) to function according to its specifications for several years at high luminosity $\left(10^{34} \mathrm{~cm}^{-2} \mathrm{~s}^{-1}\right)$.

The main concerns of our studies have been:

- The charged particle, neutron, and photon fluxes, and the hit rates generated in the inner tracker and the muon spectrometer: We have developed appropriate shielding configurations to reduce the neutron, photon, and charged particle fluxes in the detector. Sections 3-5 discuss the major sources of background and candidate configurations for shielding the muon system and the central detector cavity. As detailed in Reference 1, we applied the fluences ${ }^{\dagger}$ and energy spectra to the various detector systems and electronics to estimate the hit rates and effects on trigger rates and pattern recognition. The singles rates, trigger rates, and data flow were kept within the design limits of the data acquisition system. The occupancies in the inner tracker and in the muon spectrometer were below the limit where the pattern recognition efficiency, particle identification efficiency, or track reconstruction would be substantially degraded. For the muon system, neutron and photon fluxes less than $10^{11} / \mathrm{cm}^{2} / \mathrm{SSCY}$ led to low trigger rates and robust pattern recognition efficiency.

- The radiation doses delivered, especially to the inner tracker layers, the endcap and forward calorimeter components, and the beam line components within and near the experiment: For the fast charged particle component, systems that are most likely to suffer radiation damage are located in the central cavity, the endcap calorimeter, and the forward calorimeter. We have computed the total dose rates for detector components in these regions. The integrated fluences and doses over the life of the experiment are at levels that allow for long-term component survival. Examples of the survival limits that have dictated design choices in GEM are: 5 Mrad of ionizing dose or $10^{14} \mathrm{n} / \mathrm{cm}^{2}$, for silicon detectors; 10 Mrad for standard cables; $200 \mathrm{Mrad}$ for superinsulation; and 50 watts of thermal energy dissipated in the nearest superconducting low beta quadrupole magnet.

- Radioactivation of the inner and forward parts of the detector and the beam line, and levels of radioisotopes generated in the experimental hall: Given the close association between the calculation of particle fluences and of component activation, we have included a summary of the activation calculation procedures in Section 7. The shielding configuration and choice of materials in GEM have been designed to allow access for detector repair and maintenance operations.

While our studies so far have been geared primarily towards reducing the background of secondary neutrons and photons, we have also considered safety, cost, and access for maintenance of the detector.

It is difficult to estimate the fluences and dose rates that will occur during accelerator commissioning or under unusual accelerator conditions. Therefore, all fluences and doses presented in this chapter are normalized to a standard year $\left(10^{7} \mathrm{~s}\right)$ of running at a standard luminosity of $10^{33} \mathrm{~cm}^{-2} \mathrm{~s}^{-1}$. This corresponds to $10^{15}$ interactions produced at the interaction point, and $1.3 \times 10^{14}$ protons circulating in each ring of the collider.

TOne obtains the rate of interactions per unit volume by multiplying flux or fluence by the cross section and the target number density. 
a)

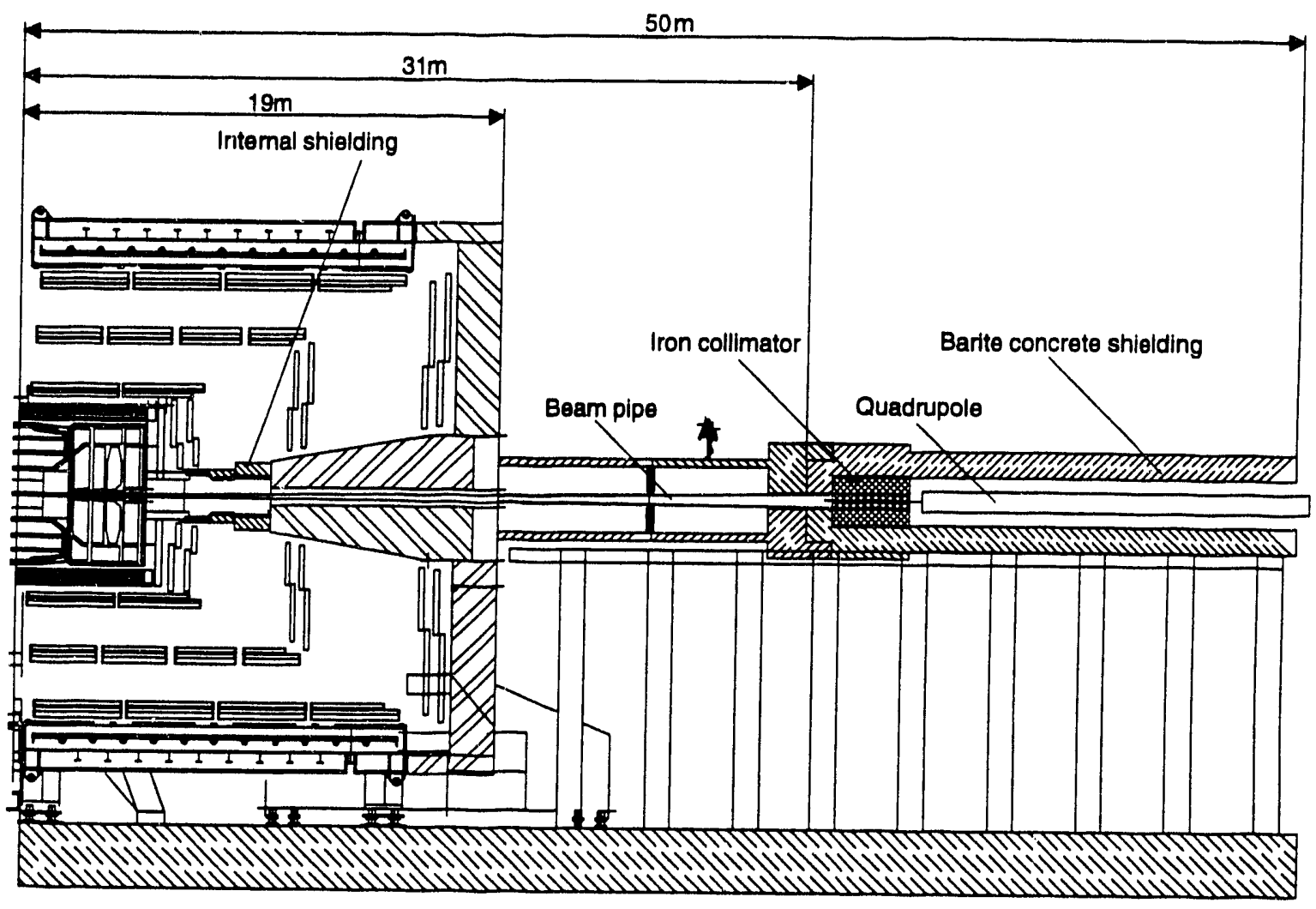

b)

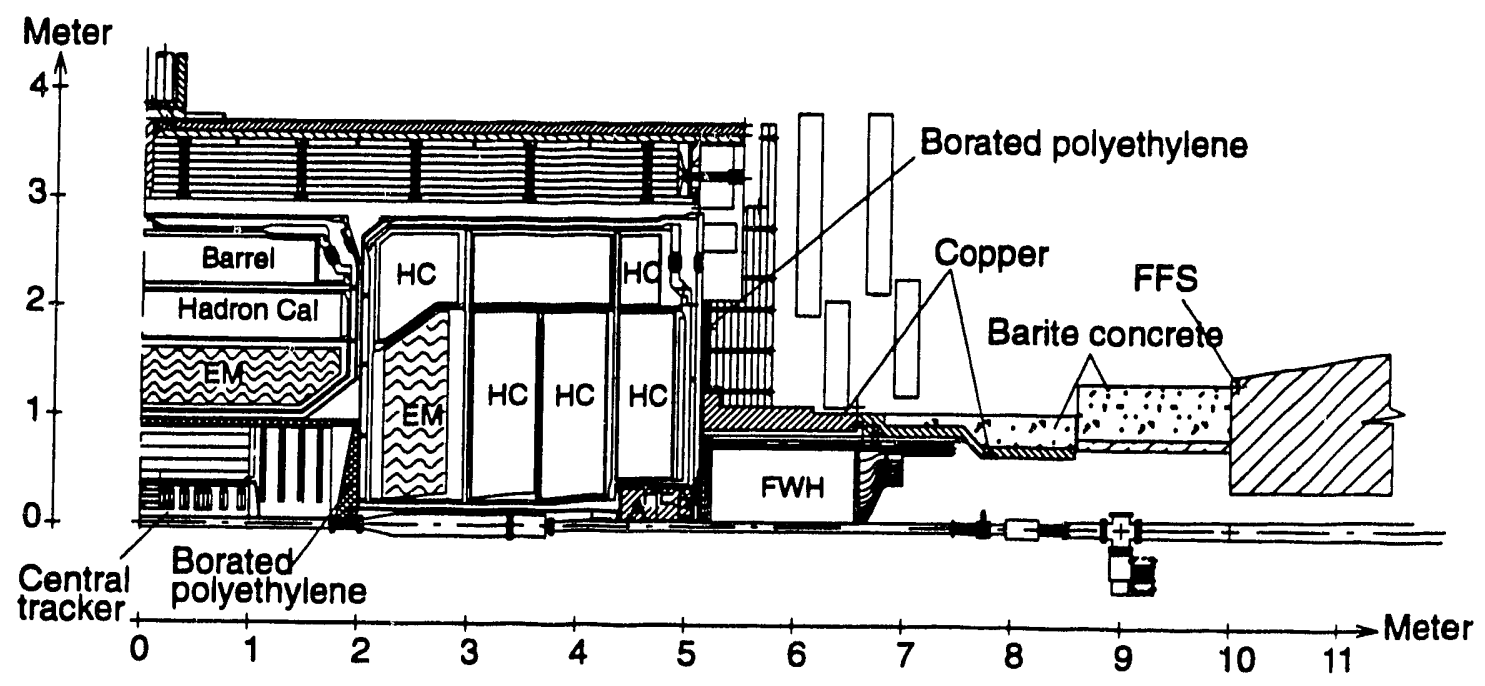

Figure 1. Detector and shlelding conflguration used in this study to calculate particle fluences and dose rates. Same calculations were also performed without the indlcated shlelding. a) Elevation view of half of the detector and shielding around the collimator and the quadrupole. b) Details of the shielding near the gem calorimeter. 
Figure $1 \mathrm{a}$ and $\mathrm{lb}$ are schematics of the detector and shielding configuration used for the results presented here. For this study we have concentrated on shielding the greatest sources of radiation in the detector: the collimator, the forward calorimeter, and the central tracker region. In the following sections we present discussion of shielding configurations and strategy for reducing particle fluxes to tolerable levels.

\subsection{COMPUTATIONAL METHODS}

We have performed this study in three stages. First, we identified the most important radiation sources by considering the deposited energy and the thickness of material between the source and the nearest sensitive detector. Second, we performed calculations to optimize the geometry and the shielding around the main sources. Finally, we performed detailed calculations of the particle fluxes, dose rates, and residual activity. To make the calculations, we used the following simulation tools.

- DTUJET: This is a Monte Carlo program for hadronic multi-particle production in $40 \mathrm{TeV}$ pp collisions. It is based on the two component dual parton model which treats the single diffractive component, soft (low $p_{T}$ ), and hard (minijet and large $p_{T}$ ) processes in a unified and consistent way. ${ }^{2}$ We use this model in all simulations to give us the distribution of particles from $40 \mathrm{TeV} p p$ interactions.

- MARS12: This is a Monte Carlo program for three-dimensional simulations of hadronic and electromagnetic cascades and muons. The program emphasizes inclusive particle production and statistical weighting, and therefore allows fast simulations. Versions of the code that interface with magnetic fields and accelerator transport exist, making this system particularly well suited for accelerator shielding studies. ${ }^{3}$

- LAHET-MCNP: This Monte Carlo code package, developed at Los Alamos National Laboratory, ${ }^{4}$ provides transport of hadrons through a combination of the FLUKA code and an extended version of the HETC code to less than $1 \mathrm{MeV}$ for charged hadrons and $20 \mathrm{MeV}$ for neutrons. MCNP transports neutrons down to thermal energies; electro" magnetic processes are handled by ITS or EGS. This code system is widely used for shielding designs of accelerators and nuclear reactors. We have not implemented the magnetic field options in LAHET for the studies described here.

- CALOR: A hadronic shower simulation package developed at Oak Ridge National Laboratory, ${ }^{5}$ CALOR combines HETC, which uses the high energy fragmentation model from FLUKA and an intranuclear-cascade evaporation model at low energies; SPECT, which analyzes information from HETC; MORSE, which transports neutrons with kinetic energy below $20 \mathrm{MeV}$; and EGS, which transports electrons and photons.

- GEANT: This code package is widely used in high energy physics. ${ }^{6}$ The main advantages of GEANT are the ease of geometry specification and accurate tracking through magnetic fields. For this study we used our implementation of GEANT containing a complete description of the GEM detector; this code package is called SIGEM. ${ }^{7}$ Our version of GEANT uses CALOR for low energy hadron transport. ${ }^{8}$

- CINDER: This code, developed at Los Alamos National Laboratory, ${ }^{9}$ computes residual radioactivity. It uses the spallation products of nuclei produced in the LAHET Monte Carlo code and the low energy neutron spectra produced by MCNP to predict nuclide density, activity, decay heating from electrons and photons, and photon spectra. These data are further processed by LAHET to provide dose rates at specified locations and times.

These tools were used for different tasks, and consistency checks were made between the different simulations and simple hand calculations using scaling models. The scaling laws from the literature $10,11,12,13$ were used for many of these checks. 
The radiation environment in the GEM detector differs in many ways from the environment described in an earlier SSC Laboratory Central Design Group report, SSC-SR-1033. ${ }^{10}$ This report concentrated on the radiation environment in the central detector cavity; these considerations are still valid, and we use the results extensively in our report. However, the radiation environment in the muon system outside the calorimeter and, in particular, the albedo from the collimators and the hall walls was not considered there. We devote much of our discussion to reducing neutron and photon fluences in the muon system because this is the more difficult problem.

\subsection{ENERGY DEPOSITION}

There are three major sources of radiation: local beam loss in the collider tunnels; interactions of the $20 \mathrm{TeV}$ proton beams with the residual gas in the beam pipe; and particle production at the interaction point. The contributions of each of these sources to the total particle fluence and the deposited energy are presented in the following sections. The radiation levels are dominated by the particle production at the interaction point.

\subsection{Contribution by Protons Lost from the Beam}

Beam loss in the walls of the beam pipe occurs continuously around the accelerator. The beam size is large in the final focus quadrupoles near the interaction points (IP), so the beam loss is particularly severe in these regions. ${ }^{13}$ Using the MARS12 code system we have estimated neutron fluxes due to this beam loss by making simulations of proton orbits around the accelerator and of the hadronic cascades due to the lost protons. The maximum loss of about $5 \times 10^{4}$ protons $/ \mathrm{m} / \mathrm{s}$ occurs at the points of maximum beta about $70 \mathrm{~m}$ from the interaction point. Figure 2 shows the neutron fluence for neutron energy between $0.5 \mathrm{eV}$ and $14 \mathrm{MeV}$ caused by beam loss as a function of the distance from the interaction point, and at a radius of $200 \mathrm{~cm}$ from the beamline. The calculation includes the tunnel and hall walls but does not include the GEM detector. Hadrons with kinetic energy greater than $14 \mathrm{MeV}$ contribute less than $10 \%$ of the total particle fluence. There is a large increase of the neutron fluence in the tunnel that starts $55 \mathrm{~m}$ from the interaction point; however, near the detector the fluences are less than $10^{9} \mathrm{n} / \mathrm{cm}^{2} / \mathrm{SSCY}$, which is negligible compared to the contribution from $p p$ interactions shown below.

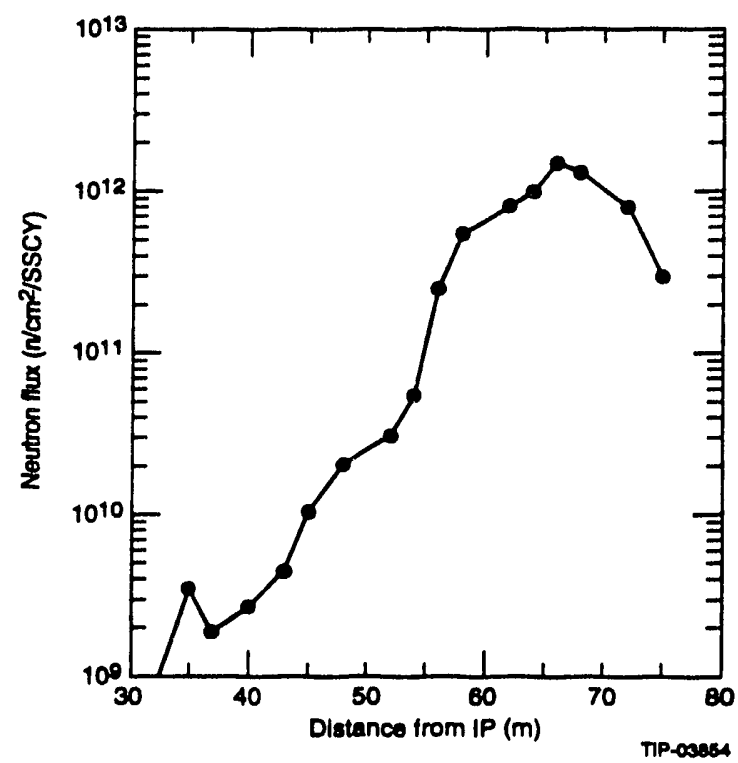

Figure 2. Neutron fluence due to local beam loss in the low beta quadrupoles as a function of distance from the interaction point for a radlus of $200 \mathrm{~cm}$ from the beamline (from a MARS12 calculation). The curve is for neutrons with klnetlc energy between $0.5 \mathrm{eV}$ and $14 \mathrm{MeV}$. Hadrons with kinetlc energy greater than 14 mev contribute less than $10 \%$ of the total particle fluence. 


\subsection{Contribution from Residual Gas in the Beam Pipe}

The particle fluence due to interactions of $20 \mathrm{TeV}$ beam protons with atoms of residual gas (beam gas interactions) in the evacuated beam pipe was computed using the MARS12 code system. The calculation assumed a pressure of $10^{-8}$ torr of nitrogen in the warm regions of the beam pipe (which extends up to the low beta quadrupoles located $35 \mathrm{~m}$ from the interaction point). A density of $4.0 \times 10^{8} \mathrm{~N}_{2}$ molecules $/ \mathrm{cm}^{3}$ was assumed for the cold regions of the beam pipe inside the magnets. The cross section of $20 \mathrm{TeV}$ protons ( $50 \mathrm{mb} /$ nucleon) is such that this density corresponds to a loss of $1.7 \times 10^{11} \mathrm{protons} / \mathrm{m} / \mathrm{SSCY}$ from the two rings in the $100-\mathrm{m}$-long experimental hall. We used the value of $2 \times 10^{11}$ protons $/ \mathrm{m} / \mathrm{SSCY}$, which includes a small contribution from protons whose orbits are disturbed by the other interaction regions. ${ }^{1}$

The results are shown in Figure 3, which gives the particle flux due to beam gas interactions in the shielded GEM detector for all hadrons with energy greater than $0.5 \mathrm{eV}$. There is a large increase in the forward calorimeter region due to the showering of secondary particles produced in the beam gas interactions. Table 1 compares the contribution from beam gas interactions at selected locations in the detector to the fluences due to $p p$ collisions for both the shielded and the unshielded detector configuration. We divide the particle flux in Table 1 into high energy $(E>14 \mathrm{MeV})$ neutrons and charged hadrons and low energy $(0.5 \mathrm{eV}<E<14 \mathrm{MeV})$ neutrons. The relative contribution from beam gas interactions in the muon system is small, and the shielding does not affect it significantly. In the central detector cavity beam gas interactions contribute $6 \%$ of the high energy particle flux at the inner silicon layers. The neutron contribution from beam gas interactions is small, with or without shielding. Our conclusion is that the attainable vacuum in the detector region could be important. Were it to become ten times worse than we have assumed, the particle flux from beam gas interactions in the central cavity would become significant. This would cause an unacceptable degradation in the lifetime and performance of the silicon tracker.

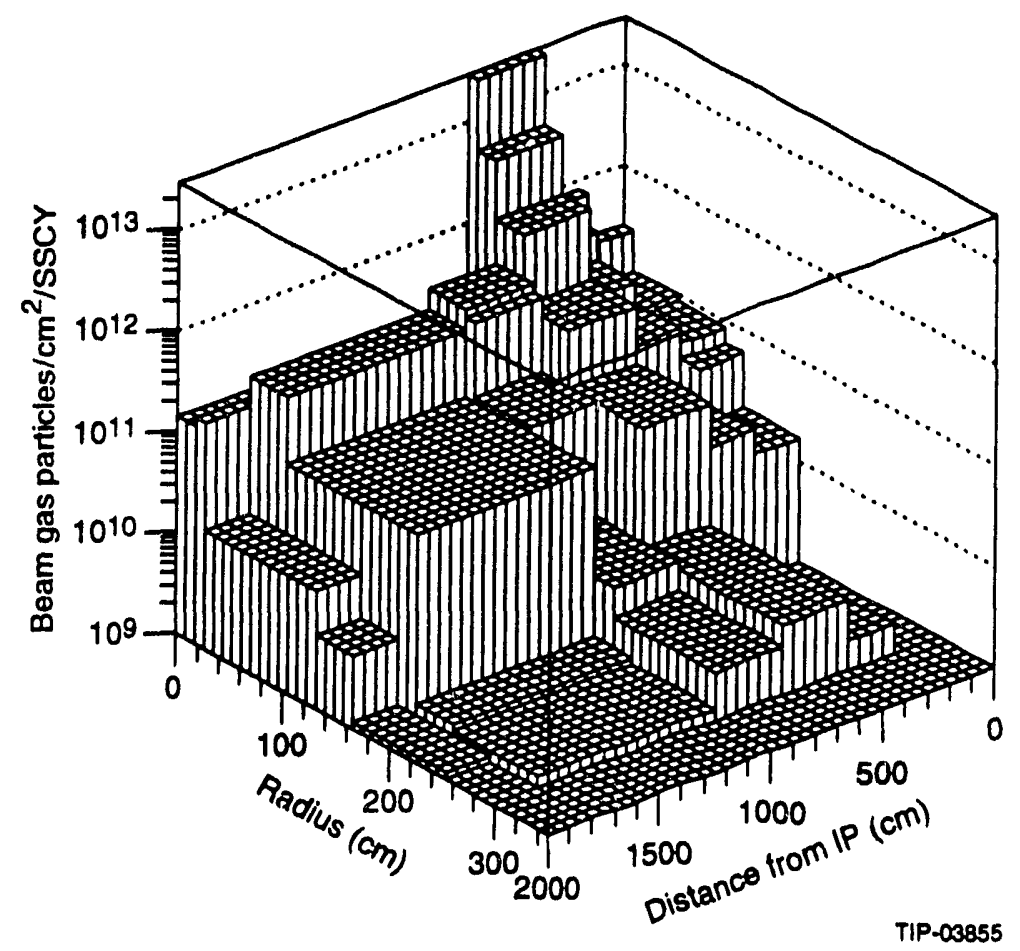

Figure 3. Particle flux due to $2 \times 10^{11}$ beam gas interactions/m/SSCY calculated using the MARS12 code system for all hadrons with energy greater than $0.5 \mathrm{eV}$. We have chosen bins of variable size in order to Illustrate the main features of the distribution. 
Table 1. Ratio in percent of the particle flux caused by beam gas interactions to the flux from pp collisions, at selected locations in the detector. This calculation was performed with MARS12 for the comparison of the two contributions.

\begin{tabular}{|c|c|c|c|c|c|c|}
\hline \multirow[t]{3}{*}{ Location } & \multirow[t]{3}{*}{$\begin{array}{l}\text { Radius } \\
\text { moter }\end{array}$} & \multirow[t]{3}{*}{$z$ from IP } & \multicolumn{4}{|c|}{$\begin{array}{c}\text { Beam gas/pp collisions \% } \\
\text { hadrons with } E>14 \mathrm{MeV} \text { and } \\
\text { neutrons with } 0.5 \text { eV }<\text { E<14 MeV }\end{array}$} \\
\hline & & & \multicolumn{2}{|c|}{ Unshielded Detector } & \multicolumn{2}{|c|}{ Shielded Detector } \\
\hline & & & $E>14 \mathrm{MeV}$ & $E<14 \mathrm{MeV}$ & $E>14 \mathrm{MeV}$ & $E<14 \mathrm{MeV}$ \\
\hline $\begin{array}{l}\text { Central } \\
\text { Tracker }\end{array}$ & $\begin{array}{l}0.06 \\
0.30 \\
\end{array}$ & $\begin{array}{l}0.0 \\
0.0\end{array}$ & $\begin{array}{l}5.8 \\
2.3 \\
\end{array}$ & $\begin{array}{l}1.1 \\
2.2 \\
\end{array}$ & $\begin{array}{l}5.8 \\
2.3 \\
\end{array}$ & $\begin{array}{l}2.4 \\
3.0 \\
\end{array}$ \\
\hline $\begin{array}{l}\text { Forward } \\
\text { Calorimeter }\end{array}$ & 0.05 & 5.5 & 0.9 & 1.0 & 0.6 & 0.9 \\
\hline $\begin{array}{l}\text { Muon } \\
\text { System }\end{array}$ & $\begin{array}{l}1.40 \\
2.50\end{array}$ & $\begin{array}{r}8.5 \\
16.0\end{array}$ & $\begin{array}{l}2.0 \\
1.6\end{array}$ & $\begin{array}{l}4.8 \\
3.6\end{array}$ & $\begin{array}{l}0.8 \\
8.2\end{array}$ & $\begin{array}{l}3.6 \\
2.2\end{array}$ \\
\hline
\end{tabular}

\subsection{Contribution from Collisions at the Interaction Point}

We used the event generator DTUJET to estimate the energy deposited in the detector and in the nearby beamline elements. Figures 4 and 5 show the pseudorapidity and transverse momentum distributions, respectively, of charged particles produced by this model. Figure 6 shows the fraction of the total energy from the interaction point emitted as a function of pseudorapidity. Table 2 summarizes the fraction of total energy deposited in the various components of the GEM detector.

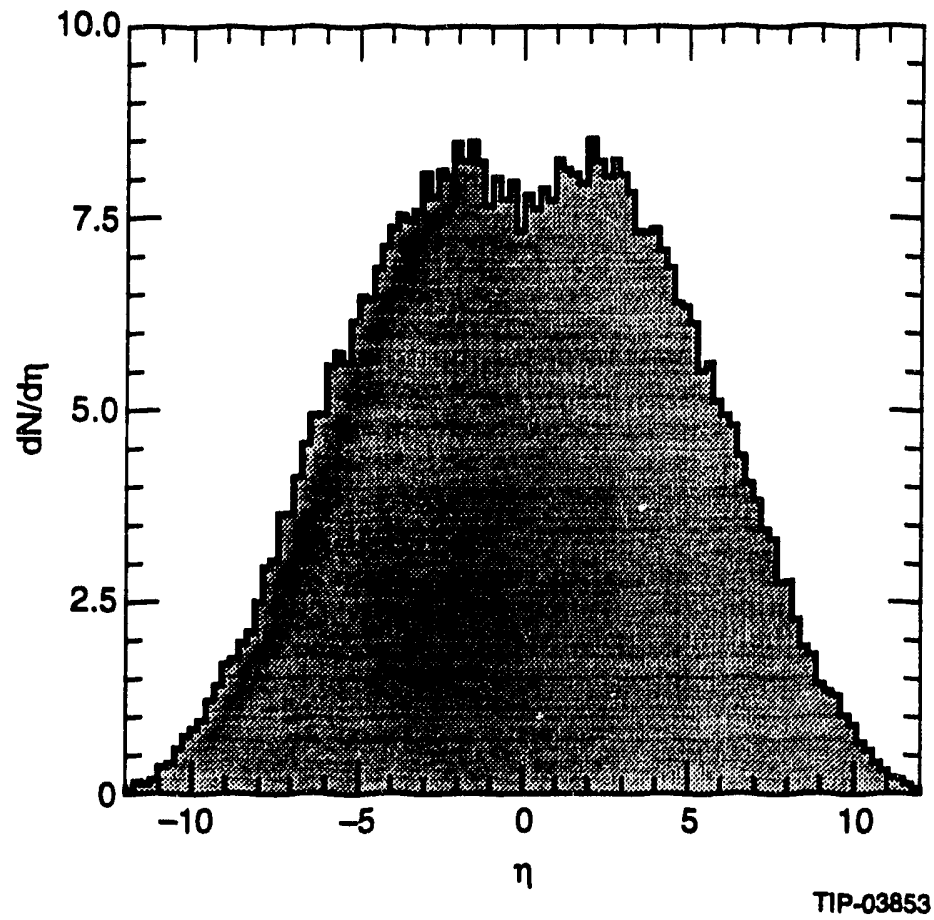

Figure 4. dN/ $\eta$ distribution for charged particles produced by the Monte Carlo code DTUJET for 40 TeV pp collisions. The plateau is approximately 7.5 charged particles per unit pseudorapidity. The dip at $\eta=0$ is due to a kinematic effect, and corresponds to a flat distribution in rapidity. 


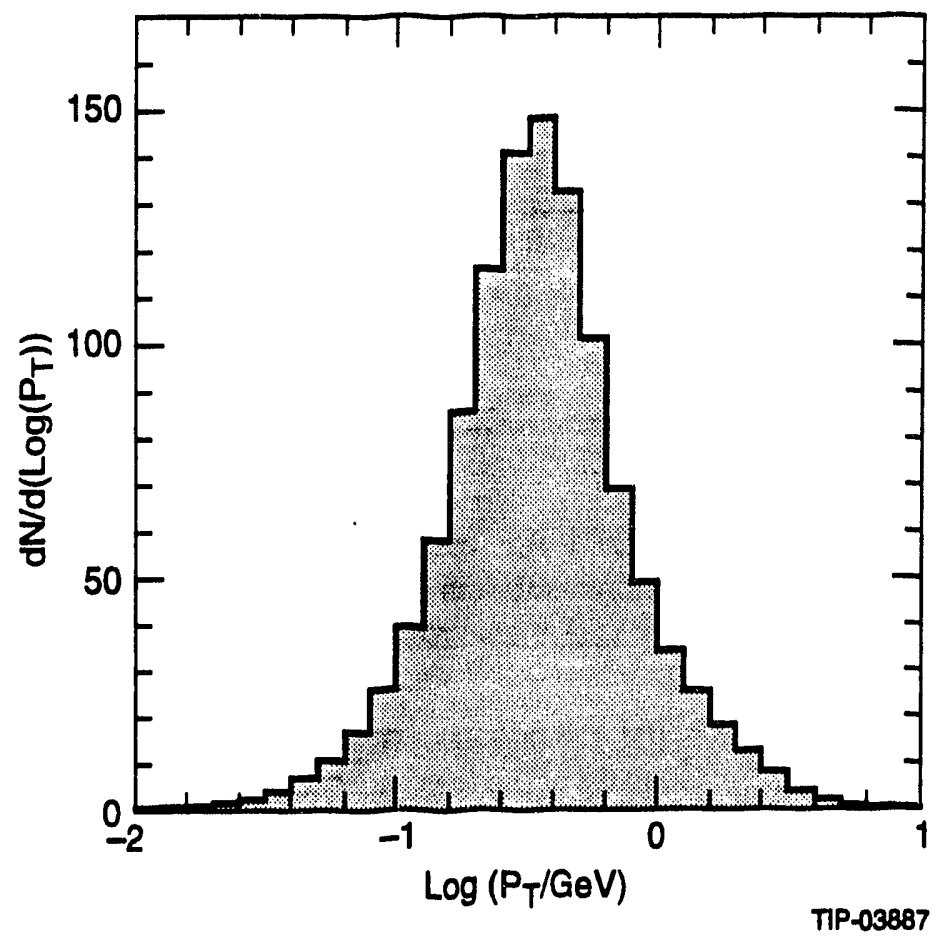

Figure 5. $d N d p$ distribution for charged particles produced by the Monte Carlo code DTUJET for $40 \mathrm{ToV}$ pp collision. The mean of the distribution ls approximately $0.6 \mathrm{GeV}$.

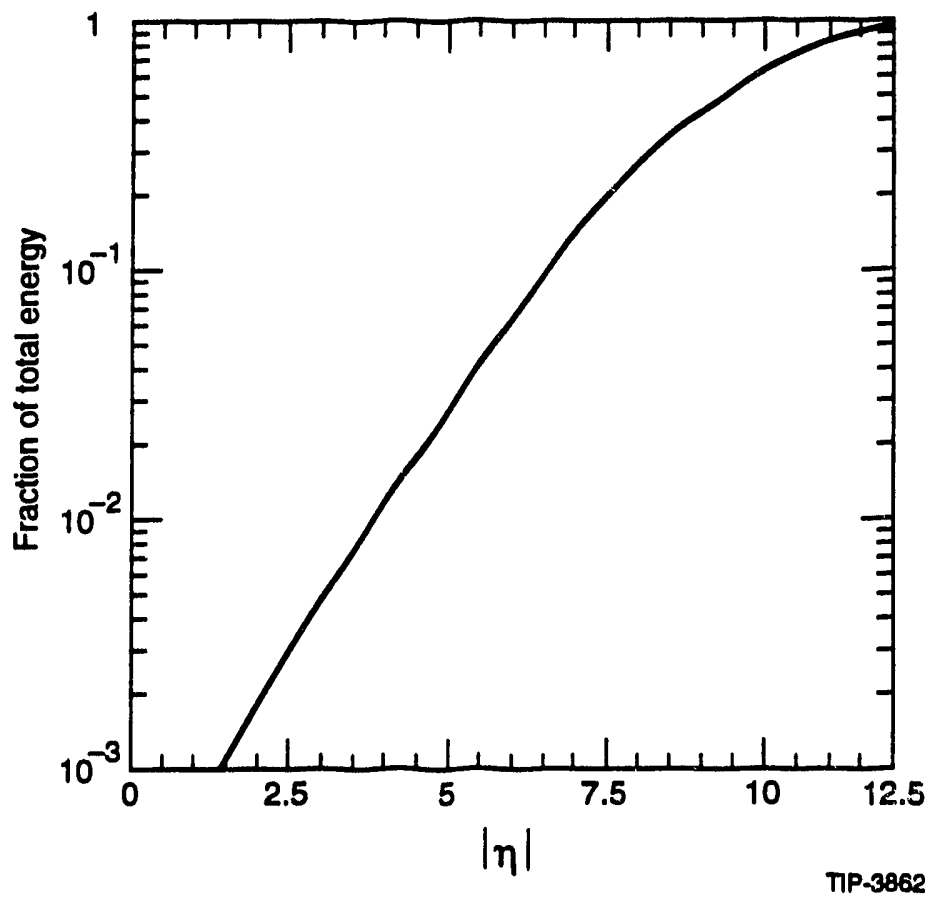

Figure 6. Fraction of the total energy from the interaction point emitted as a function of pseudorapidity. 
Table 2. Fraction of the total energy emitted from the interaction point intercepted by various detector components.

\begin{tabular}{|l|c|c|}
\hline \multicolumn{1}{|c|}{ Detector } & $\eta$ Coverage & $\begin{array}{c}\text { \% Energy } \\
\text { Deposited }\end{array}$ \\
\hline Barrel calorimeter & $0<|\eta|<1.3$ & 0.1 \\
Endcap calorimeter & $1.3<|\eta|<3.0$ & 0.4 \\
Forward calorimeter & $3.0<|\eta|<5.8$ & 4.4 \\
Collimator & $5.8<|\eta|<8.6$ & 27.0 \\
\hline
\end{tabular}

The pseudorapidity plateau of 7.5 charged particles per unit pseudorapidity and mean transverse momentum of $0.6 \mathrm{GeV}$ is in agreement with extrapolation of data from lower energies. There is no data in the far forward regions $(|\eta|>6.0)$; therefore, we assign an error of a factor of 2 to the distribution of total radiated energy. ${ }^{15}$

An iron collimator covering $5.8<|\eta|<8.6$ is placed in front of the quadrupoles to intercept energy that would otherwise go into the cryogenic mass of the low beta quadrupoles. The amount of energy lost from the collider at the interaction point $\left(4.0 \times 10^{9} \mathrm{TeV} / \mathrm{s}\right)$ is far greater than the beam loss in the accelerator magnets near the interaction point (approximately $4.0 \times 10^{7} \mathrm{TeV} / \mathrm{s}$ ) or the beam loss in the residual gas of the vacuum pipe $\left(4.0 \times 10^{7} \mathrm{TeV} / \mathrm{s}\right)$. The energy intercepted by the forward calorimeters $\left(1.8 \times 10^{8} \mathrm{TeV} / \mathrm{s}\right)$ is much less than the energy into the collimators $\left(1.1 \times 10^{9} \mathrm{TeV} / \mathrm{s}\right)$. Nevertheless, due to the proximity of some muon chambers to the forward calorimeter, both the collimator and the forward calorimeter regions will be the dominant sources of background neutron and photon fluences in the muon system of the detector. The entrance to the central cavity presents a very small solid angle to the forward calorimeter and the collimator; we therefore expect the fluxes in the central cavity to result largely from the albedo of electromagnetic and hadronic showers in the barrel and endcap calorimeters.

Figure 7 shows neutron and photon fluxes for neutrons of all energies for an unshielded detector (calculated using the GEANT-CALOR package). The flux in the central tracker is seen to be uniform at a level of $5 \times 10^{13} \mathrm{n} / \mathrm{cm}^{2} / \mathrm{SSCY}$; the flux in the muon system varies between $10^{11}$ and $10^{12} \mathrm{n} / \mathrm{cm}^{2} / \mathrm{SSCY}$. Figure 8 shows the two main components of the neutron flux: the collimators and the calorimeter. Sections 4.1 to 4.4 discuss shielding that can be built around the collimators and the forward calorimeter. Shielding concerns for the central tracker are addressed separately in Section 5. 

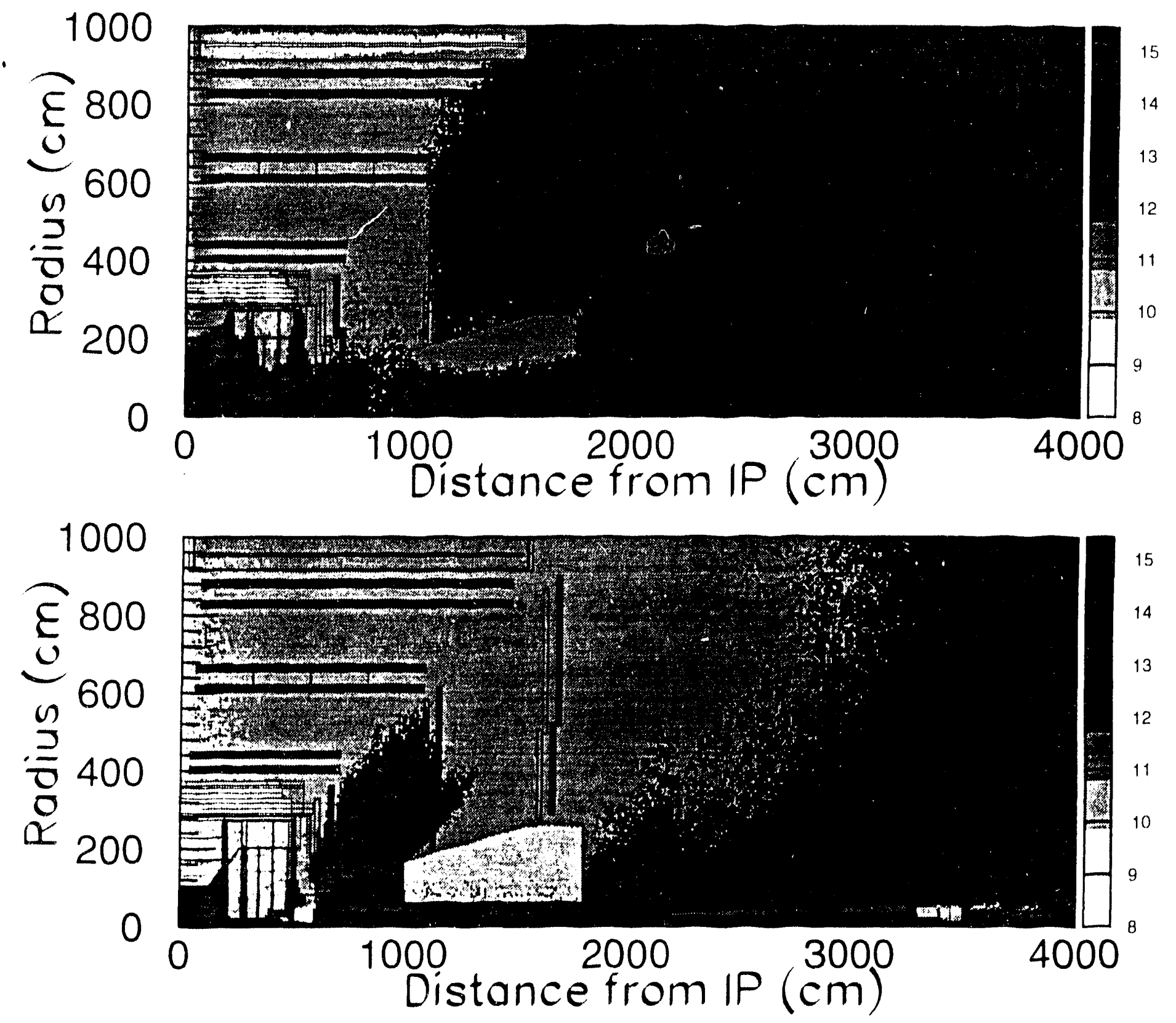

Figure 7. Neutron (n) and photon $(\gamma)$ flux, in units of $10^{n} / \mathrm{cm}^{2} / \mathrm{SSCY}$, where the color (or shade) Indicates the power $n$, in the unshielded detector (calculated using the GEANT-CALOR package).

Neutrons of all energies are included in the plot; photons have an energy cutoff $E>10 \mathrm{keV}$. 

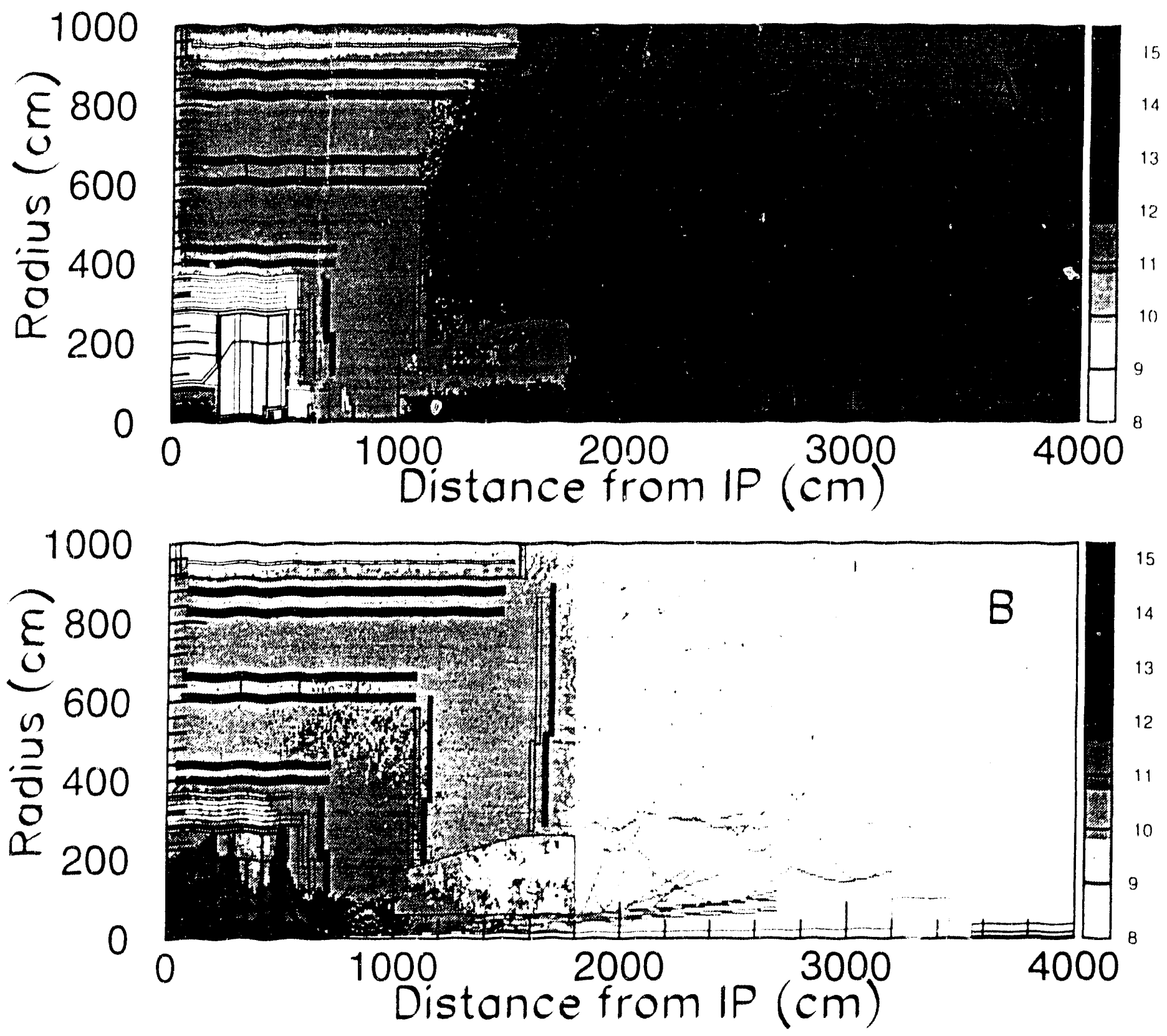

Figure 8. Neutron flux, in units of $10^{n}$ neutrons $/ \mathrm{cm}^{2} / S S C Y$, where the color (or shade) indicates the power $n$, from $A$ ) Interactions in the collimator, quadrupoles, and beam line elements, B) Interactions in the calorimeter system. 


\subsection{SHIELDING CONSIDERATIONS}

\subsection{Location and Aperture of the Collimators}

We determined the location and the inner diameter of the collimator, taking into consideration the amount of energy deposited in the low beta quadrupoles and the neutron fluences in the detector. For fixed aperture sizes, moving the collimator away from the interaction point reduces the solid angle for particles impinging on the cold magnets. Nevertheless, the energy going into the magnets is a weak function of the collimator distance to the interaction point, because the distribution of energy from the interaction point is extremely forward-directed (Figure 6). For example, with a collimator inner diameter of $25 \mathrm{~mm}$, moving the collimator from $20 \mathrm{~m}$ to $35 \mathrm{~m}$ reduces the energy going into the quadrupoles by cnly $20 \%$. The theoretical uncertainty in the forward production is much larger than this. The neutron flux in the detector from hadronic showers in the collimators is also a weak function of the collimator location. This is because a significant fraction of the radiation from the collimator undergoes secondary reactions in the concrete walls of the collision hall and the forward regions of the detector (see Figure 8), reducing the effect of the collimator as a localized source. Therefore, the location of the collimator and first quadrupole is largely determined by considerations of luminosity, space needed for shielding the collimators, and detector access. We have determined that placing the first low beta quadrupole so that it starts $35 \mathrm{~m}$ from the interaction point is a reasonable choice. A location closer to the interaction point might lead to a modest increase in luminosity, but the increase would most likely be offset by the increased amount of time needed during accesses for removing shielding.

A fraction of the energy going into the low beta quadrupoles appears as heat deposited in the cold mass of the magnets. Table 3 shows the amount of heat deposited in the cold mass of the first quadrupole and the expected lifetime of the coil insulation based on a $1000 \mathrm{Mrad}$ limit. These quantities are tabulated as functions of the inner diameter of a 3-m-long iron collimator starting $32 \mathrm{~m}$ from the interaction point. Using the MARS12 program, these numbers were calculated by simulating the showers induced by particles coming from the interaction point and striking the collimator-quadrupole system. The magnetic field in the quadrupoles was included in the simulation. The field approximately doubles the amount of heat deposited in the first quadrupole by bending forward-going particles that would otherwise have deposited their energy farther downstream. There is a large uncertainty on the heat load due to the uncertainties in the DTUJET model. The acceptable heat load for the first quadrupole (determined by the SSC magnet group) is approximately 50 watts. We propose a collimator with a $25 \mathrm{~mm}$ diameter aperture. Finally, the present design of the low beta quadrupoles with maximum acceptable heat load of 50 watts is expected to be upgraded along with several subsystems of the SSC accelerator. This will allow GEM to operate at a luminosity of $10^{34} \mathrm{~cm}^{-2} \mathrm{~s}^{-1}$.

Table 3. Power dissipated in the first low beta quadrupole and the expected lifetime of the coil Insulation at standard luminosity $\left(10^{33} \mathrm{~cm}^{-2} \mathrm{~s}^{-1}\right)$ as functions of the collimator aperture.

\begin{tabular}{|l|l|l|l|}
\hline Aperture (mm) & 25 & 12 & 300 \\
Heat in QL1 (watts) & 21 & 40 & 85 \\
Lifetime (year) & 7 & 5 & 1.5 \\
\hline
\end{tabular}

\subsection{Shielding for the Collimators}

The spectrum of particles striking the iron collimator peaks at about $100 \mathrm{GeV}$, but particles with energies of several $\mathrm{TeV}$ are also present. We expect the 3-m thick iron (16.7 interaction lengths) collimator to absorb most of the high energy radiation resulting from the hadronic cascade without significant leakage into the cold mass of the low beta quadrupole magnet. At the end of the hadronic cascade in dense material, only neutrons with kinetic energies of 1 to $20 \mathrm{MeV}$ from nuclear evaporation remain. Multiple neutron production 
and inelastic $\left(n, n^{\prime}\right)$ reactions are important in this energy range. Although these processes generate extra neutrons, they are effective in degrading the neutron energies. As the neutron energy is degraded below a hundred $\mathrm{keV}$, the most effective neutron removal process is the $(\mathrm{n}, \gamma)$ reaction, where a neutron is captured with the emission of one or more photons with energy below a few MeV. Unfortunately, $(n, \gamma)$ cross sections are not high enough in most heavy metals to rapidly remove neutrons below a hundred $\mathrm{keV}$. ${ }^{16,17}$ This is especially true for iron and lead. Therefore, neutrons in the 0.1 to $1.0 \mathrm{MeV}$ range are expected to leak out of the collimator into the hall. We are forced to use light materials such as borated polyethylene or borated concrete with high hydrogen content to rapidly thermalize these neutrons and absorb them on boron or other nuclei that have very high absorption cross sections at thermal energies. We expect that the best shields will be composites made of heavy metals to contain the high energy cascades in a small volume, and light materials such as concrete or polyethylene to moderate and absorb the low energy neutron leakage.

We have investigated several different geometrical arrangements of concrete around the collimator and have found the neutron flux attenuation largely insensitive to the geometry when at least $5 \mathrm{~m}$ of concrete is present to trap the albedo from the collimator, which is located at $32 \mathrm{~m}$ from the interaction point. Table 4 shows the flux of neutrons in the $0.5 \mathrm{eV}$ to $14 \mathrm{MeV}$ range in various parts of the detector for two different configurations of shielding around the collimator:

Table 4. Neutron fluxes at specified locatlons in the $0.5 \mathrm{eV}$ to $14 \mathrm{MeV}$ range for two different configurations of shlelding around the collimator (see text for the exact geometry). The second configuration has a 5-m-long albedo trap in front of the collimator, additional concrete around the collimator, and $1 \mathrm{~m}$ of concrete around the low beta quadrupole magnets. The hydrogen and boron content in the concrete removes thermal neutrons effectively.

\begin{tabular}{|l|c|c|c|c|}
\hline \multicolumn{1}{|c|}{ Location } & $\begin{array}{c}\text { Radius } \\
\text { (meter) }\end{array}$ & $\begin{array}{c}\text { Distance from IP } \\
\text { (meter) }\end{array}$ & $\begin{array}{c}\text { n Flux Config. A } \\
\text { (n/cm²/SSCY) }\end{array}$ & $\begin{array}{c}\text { n Flux Config. B } \\
\text { (n/cm } 2 / S S C Y)\end{array}$ \\
\hline Endcap muon & 2.5 & 14 & $1.2 \times 10^{12}$ & $1.7 \times 10^{11}$ \\
Free space & 2.0 & 23 & $3.8 \times 10^{12}$ & $1.4 \times 10^{11}$ \\
Albedo trap & 2.0 & 30 & $7.1 \times 10^{12}$ & $5.4 \times 10^{10}$ \\
Collimator & 2.0 & 33 & $1.9 \times 10^{12}$ & $4.4 \times 10^{10}$ \\
\hline
\end{tabular}

A) The column for configuration (A) contains fluxes for an iron collimator with an outer radius of $1.5 \mathrm{~m}$ surrounded by $0.5 \mathrm{~m}$ of concrete. There was no additional shielding around the low beta quadrupoles and no shielding from the collimator albedo.

B) The column for configuration (B) contains fluxes for an iron collimator with an outer radius of $0.75 \mathrm{~m}$ surrounded by $1.25 \mathrm{~m}$ of concrete. In addition, the low beta quadrupoles from the back of the collimator to the turnel entrance were surrounded by $1.0 \mathrm{~m}$ of concrete, and a $5.0-\mathrm{m}$-long concrete block with an inner diameter of $0.5 \mathrm{~m}$ and an outer diameter of $2.0 \mathrm{~m}$ was placed in front of the collimator to trap the albedo.

The extra shielding in configuration (B) obtains a one order of magnitude reduction of the flux in the muon chambers, and a two orders of magnitude reduction between the detector and the collimator. There is also a large reduction around the collimator at $33 \mathrm{~m}$ due to the replacement of some of the iron with concrete.

The largest reduction of neutron fluxes in the detector volume results from the addition of the albedo trap. We also find that the use of special concretes such as barite ${ }^{18}$ with high barium and boron contents leads to shielding designs with low volumes. We have chosen the configuration of shielding shown in Figure 1, which gives an estimated two orders of magnitude reduction in neutron fluxes on the boundary of this shield. In addition, we have chosen to extend the shield up to the iron field shaper to eliminate gaps and trap all of the albedo. This choice should help us shield against beam gas interactions in the beam pipe as well. 


\subsection{The Forward Calorimeter}

We have applied the same general considerations to the forward calorimeter shielding as were applied to the collimator shielding. The location of this shielding in the middle of the sensitive muon system, and the function of the forward calorimeter as an active detector, present special constraints on this shielding design. In particular, we identify two separate escape paths for neutrons generated in the forward calorimeter: 1) Neutrons injected into the cryostat gap between the electromagnetic and hadronic sections of the forward calorimeter can travel through the gap to a larger radius and escape into the muon system (see Figure 1 at $z=$ $4.95 \mathrm{~m}$ ). 2) High energy particles and neutrons generated at the inner edge of the hadronic calorimeter can further interact in the iron field shaper and escape into the muon system through the gap between the forward calorimeter and the field shaper (see Figure 1 between $z=6.43 \mathrm{~m}$ and $z=10.0 \mathrm{~m}$ ). There are two additional considerations for providing protection to the muon chambers at $z=6.38 \mathrm{~m}$. First, we must place sufficient high density material at the location of the forward hadronic calorimeter transverse to the beam axis to attenuate all charged particles. Second, we cannot place a neutron absorbing material such as borated polyethylene in proximity to the muon chambers, which are sensitive to the photons emitted as a result of neutron absorption on boron.

In order to quantify the number of neutrons injected into the muon system by particle interactions, we have performed Monte Carlo simulations that include the endcap calorimeter, the forward calorimeter, and the forward field shaper using the CALOR package. The particles from the interaction point were divided into three groups: 1) $2.5<|\eta|<4.4$, particles striking the lower half of the endcap and the upper half of the forward electromagnetic section; 2) $4.4<|\eta|<5.6$, particles striking the lower half of the forward electromagnetic section; 3) $5.6<|\eta|<5.8$, particles striking the inner edge of the hadronic calorimeter. Table 5 shows the number of neutrons per second escaping through the two paths due to these three particle sources. The flux escaping through the cryostat gap is approximately $25 \%$ of the total; this can be eliminated by filling the gap with heavy material. The largest source is the inner edge of the hadronic calorimeter. It can be eliminated by either placing heavy shielding between the forward calorimeter and the field shaper, or by shaping the inner edge to be projective to the interaction point. Table 5 shows that $60 \%$ of the flux can be eliminated by making the inner edge of the forward hadronic calorimeter projective.

Table 5. Categorization of neutrons of all energies injected into the endcap muon chambers per second. The source particles from the Interaction point are categorized in three $\eta$ reglons as indicated in the rows of the table. The neutrons injected in the muon system are categorized in two groups as indicated in the third and fourth columns of the table.

\begin{tabular}{|l|c|c|c|}
\hline Source of Secondaries & $\eta$ Coverage & Cryostat Gap (Hz) & FCAL-FFS Gap (Hz) \\
\hline Top of FCAL & $2.5<|\eta|<4.4$ & $1.4 \times 10^{9}$ & $9.2 \times 10^{7}$ \\
Bottom of FCAL & $4.4<|\eta|<5.6$ & $3.1 \times 10^{9}$ & $2.2 \times 10^{9}$ \\
Inner Edge of Had. FCAL & $5.6<|\eta|<5.8$ & $5.7 \times 10^{8}$ & $8.9 \times 10^{9}$ \\
\hline
\end{tabular}

The neutron flux through the cryostat gap is reduced by approximately $30 \%$ by lining the inner edge of the passive absorber with 5-cm-thick borated polyethylene. The passive absorber is made of copper and presents approximately 3.5 interaction lengths of transverse material at the location of the forward hadronic section. We have added $10 \mathrm{~cm}$ of copper and barite concrete with an average thickness of $40 \mathrm{~cm}$ between the forward hadronic section and the field shaper (see Figure $1 \mathrm{~b}$ ). This shield reduces the neutron fluxes injected into the muon system by one order of magnitude everywhere except the muon chambers adjacent to the forward hadronic calorimeter. 


\subsection{Beam Pipe Considerations}

The beam pipe is the first material structure traversed by particles from the interaction point. We have attempted to reduce the density and the thickness of the beam pipe everywhere in the interaction region in orcier to reduce the number of secondary interactions. The beam pipe in the central tracker is nominally made of $1.5-\mathrm{mm}$-thick beryllium with an inner diameter of $80 \mathrm{~mm}$. The section of the beam pipe through the endcap region is larger in diameter (inner diameter $200 \mathrm{~mm}$ ) and is composed of 2-mm thick stainless steel. The larger diameter keeps the beam pipe wall away from the low angles where the flux and energy of particles is large. It also increases the pumping efficiency of the Getter pump located in this region. The beam pipe is narrow ( $80 \mathrm{~mm}$ diameter) through the forward calorimeter and then is joined to a flared beam pipe. We have designed gate valves and other beam line instrumentation at these joints carefully so that there are no edges to intercept particles from the interaction point.

The beam pipe is flared between the back end of the forward calorimeter and the collimator, so all the beam pipe material is in the shadow of the forward calorimeter. Thus, particles from the interaction point passing through the hole in the forward calorimeter can not interact until they enter the iron collimator in front of the low beta quads. Secondary products from the showers initiated at the inner edge of the forward calorimeter are the only significant source of interactions in this section of the beam pipe. Figure 9 shows the average number of hadronic interactions in the beam pipe as a function of distance from the interaction point for a cylindrical beam pipe with a radius of $4 \mathrm{~cm}$ and for the beam pipe described in this section. The flaring of the beam pipe will reduce the number of hadronic interactions by more than a factor of 10 , with a corresponding decrease in the neutron flux, total dose rates, and activation in the region between the forward calorimeter and the collimator.

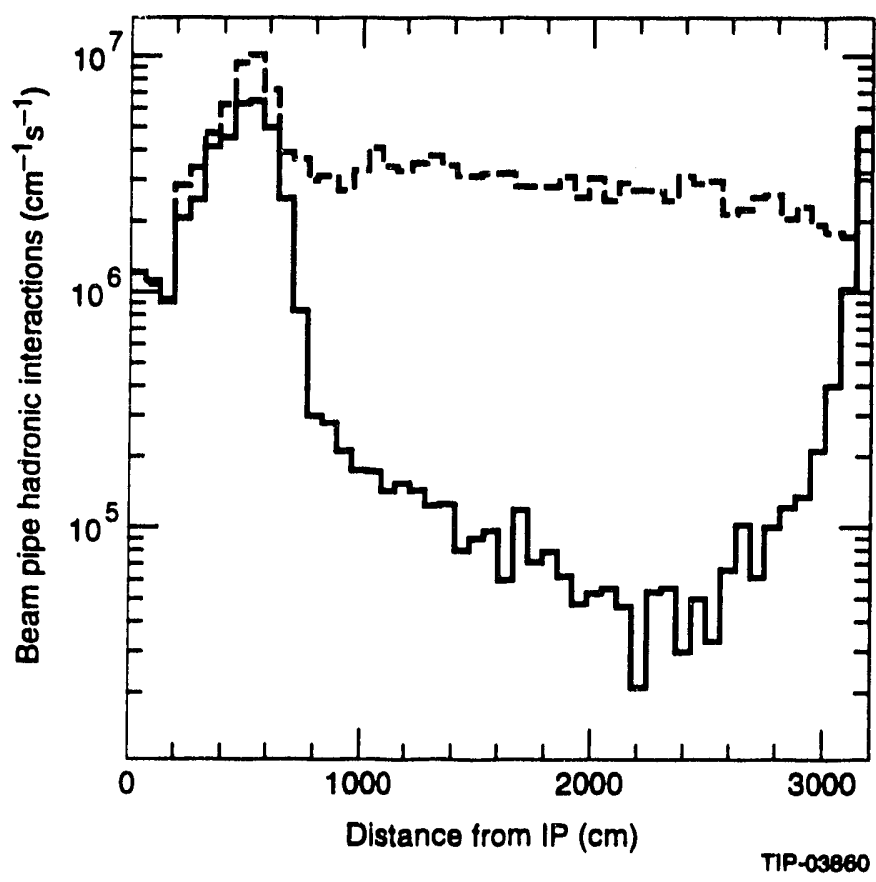

Figure 9. The average number of hadronic Interactions in the beam pipe as a function of distance from the interaction point for two cases: as described in the text (solid); and with a constant dlameter of $80 \mathrm{~mm}$ (dashed). 


\subsection{Summary of Particle Fluxes}

In this section we summarize the particle fluxes in the muon system, with and without the additional shielding described above. Fluxes in the central detector cavity are treated separately in the next section.

Charged particle fluxes are negligible outside the central detector cavity in most of the muon system. The muon flux from beam losses in the quadrupoles is also small.

The neutron fluxes for the unshielded detector are shown in Figures 7 and 8 . The main source is the collimator, as is apparent in the figures. The forward calorimeter contributes significantly to the flux at the innermost endcap muon chambers. Figure 10 shows the neutron and photon fluxes for the shielded detector. Neutrons of all energies and photons above $10 \mathrm{keV}$ are included in these figures, which were produced with the same simulation package (GEANT-CALOR). Neutron fluxes in most of the muon system are in the range of $1-5 \times 10^{10} \mathrm{n} / \mathrm{cm}^{2} / \mathrm{SSCY}$, with the exception of the muon chambers closest to the forward calorimeter where the flux is about twice as large. We expect a photon to neutron ratio of approximately $30 \%$ due to the continuous removal of neutrons by $(n, \gamma)$ reactions.

Figure 11 shows the spectra of neutrons and photons expected in the barrel muon system of the shielded detector. The spectrum of neutrons depends on the details of the shielding and the location in the detector. The thermal part of the neutron spectrum poses special problems since it is highly dependent on the water content of the air and concrete in the hall. It also depends on the presence of rare isotopes with high thermal neutron absorption cross-sections. In particular, boron in the shielding concrete should totally remove thermal neutrons in its vicinity. We have computed that less than $10 \%$ of the neutrons are thermal in the muon system of the shielded detector. Since the calculation of muon chamber hit rates, which is described in Ref. 1 and 19 , is not strongly dependent on the fraction of thermal neutrons, a thorough understanding of the thermal neutron flux is not needed. The muon chambers are approximately 10 times more sensitive to photons than to neutrons. Most of the photons in the muon system, however, are produced by interactions of neutrons. Therefore we have concentrated on removing neutrons as close to their sources (the collimator and the forward calorimeter) as possible. We have accomplished our goal by hermetically sealing the entire beamline: The thick calorimeter serves as a shield in the central region, and the concrete and the field shaper shield the beam pipe, the collimator, and the quadrupoles.

The uncertainties in this calculation result from three sources: 1) the particle production model, 2) knowledge of neutron cross sections in the materials of the detector, 3) inexact modeling of the detector geometry for computational convenience. We assign an error of a factor of 2 for the first uncertainty. The other two uncertainties are estimated by using different Monte Carlo codes. We have modeled the same fluxes using MARS 12 and the Los Alamos LAHET-MCNP code, which use different cross section libraries and tracking algorithms, and have found agreement within a factor of 2 in these codes. Therefore, we are confident that our estimates are accurate within an overall factor of 3 . The contribution to the error from Monte Carlo statistics is small on this scale.

Our results on neutron and photon fluxes are used in Ref. 1 to estimate hit rates in the muon chambers. The calculations are quite conservative. At various locations in the muon systern we compare the GEANT-CALOR and LAHET-MCNP results and always use the higher estimate for fluxes and the more pessimistic spectra. These conservative estimates of rates in the muon system are low even at the higher luminosity of $10^{34} \mathrm{~cm}^{-2} \mathrm{~s}^{-1}$. 

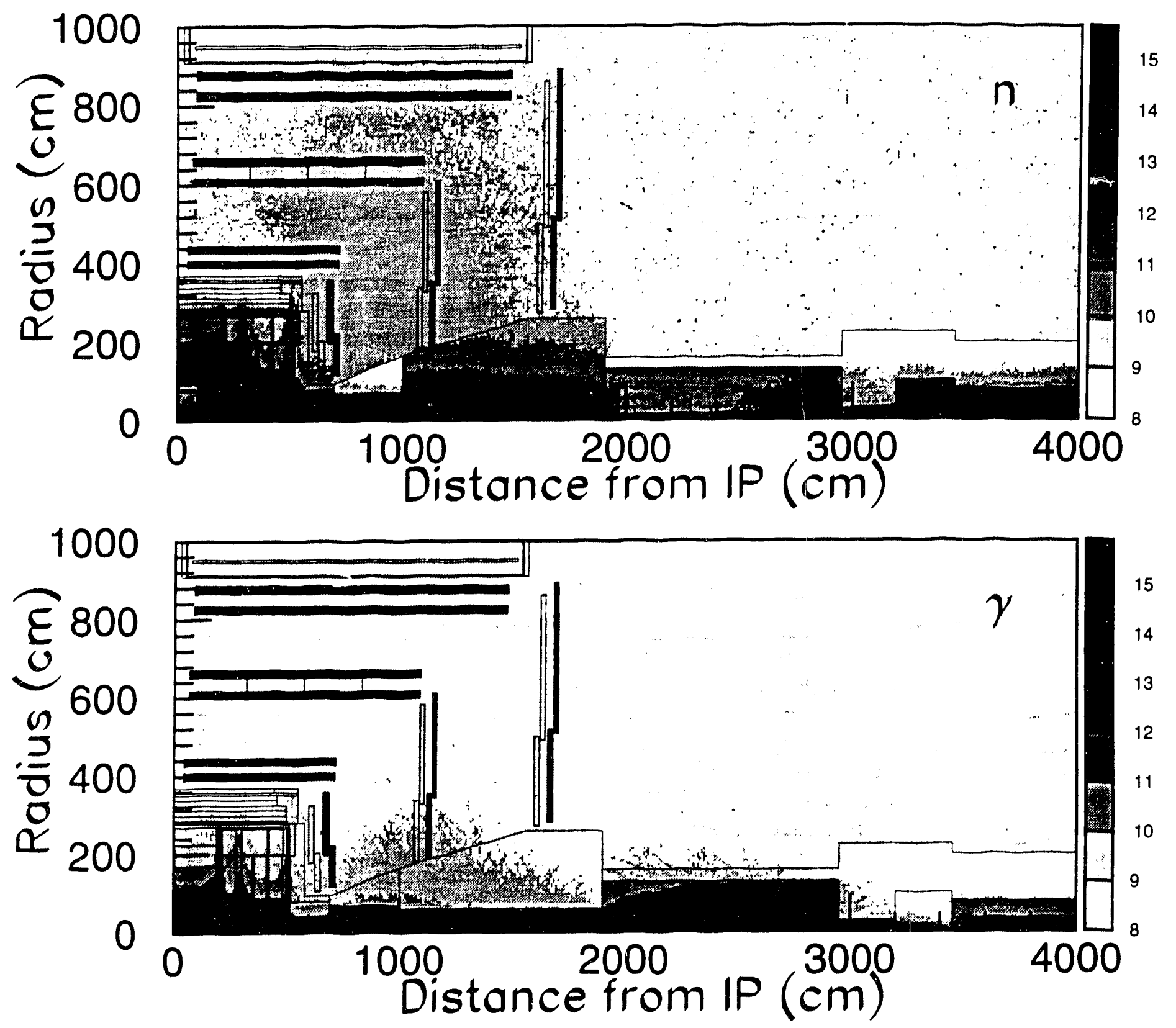

Figure 10. Neutron $(n)$ and photon $(\gamma)$ flux in the shielded detector, in units of $10^{n} / \mathrm{cm}^{2} / S S C Y$, where the color (shade) indicates the power $n$ (calculated using the GEANT-CALOR package). Neutrons of all energles are included in the plot and photons have the energy cutoff $E>10 \mathrm{keV}$. 

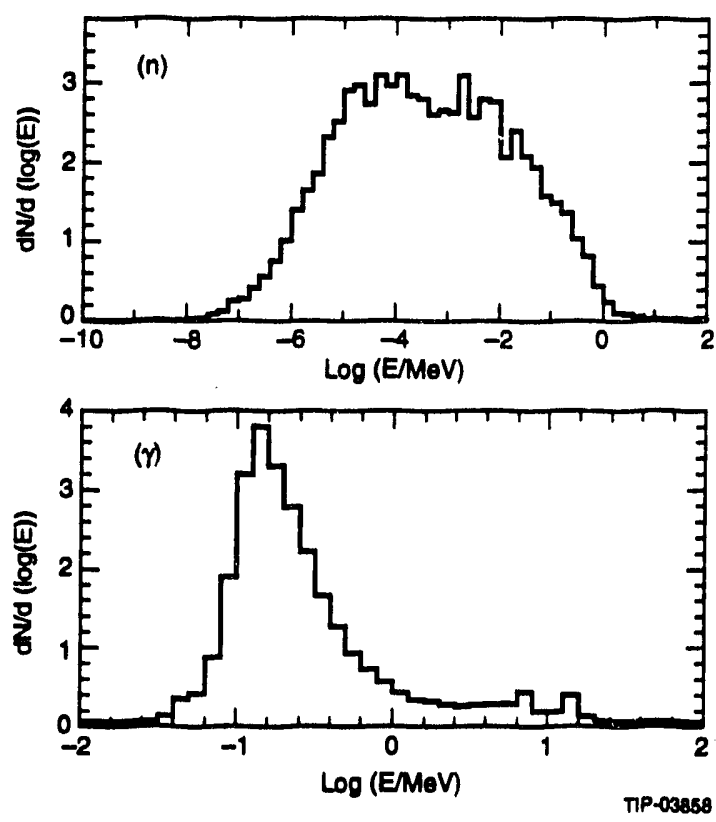

Figure 11. The spectra of neutrons ( $n$ ) and photons $(\gamma)$ in the barrel muon s;stem of the shielded detector calculated using the GEANT-CALOR package. The normallzatlon is arbltrary in these plots.

\subsection{CENTRAL TRACKER}

The albedo neutron and photon fluxes in the central cavity are affected by the volume of the cavity, the composition of the electromagnetic calorimeter, and by the shielding material placed in front of the calorimeter. We have shown that changes made in the forward calorimeter, and in the shielding of the collimators and quadrupoles, have a relatively small effect on the radiation environment in the central tracker. ${ }^{20}$ Albedo neutrons from the forward calorimeter must travel a long narrow path through the endcap; therefore they are attenuated before reaching the central cavity. As shown in Reference 10, the neutron flux scales as $1 / R^{2}$, where $R$ is the characteristic dimension of the central cavity. Furthermore, the flux is proportional to $(1+A)$, where $A$ is the mean number of reflections that a neutron experiences before being absorbed. Borated polyethylene placed on the walls of the central detector cavity should reduce the neutron flux by fast absorption. However, the reaction $\mathrm{B}^{10}(\mathrm{n}, a) \mathrm{Li}^{7}$ and subsequent decay of $\mathrm{Li}^{7}$ through photon emission $(0.477 \mathrm{MeV})$ can increase the photon flux in the central detector cavity. Therefore, we have performed calculations of neutron and photon fluxes for different amounts of borated polyethylene to understand the relative sizes of these effects.

The calculations were performed using the LAHET-MCNP code system with no magnetic field. Omission of the GEM magnetic field from the simulation introduces a negligible error to our calculations. ${ }^{10}$ Figures 12 and 13 show the neutron and photon fluxes along surfaces bordering various central tracker components. Neutrons above $100 \mathrm{keV}$ kinetic energy are far more damaging to silicon than lower energy neutrons, due to the sudden rise in the displacement cross section. ${ }^{21,22}$ Therefore, we display neutron fluxes above and below this threshold. The different curves correspond to different configurations of the borated polyethylene. The plots show that the neutron flux is relatively constant throughout the central tracker volume. It increases by a factor of approximately 1.5 in the forward regions near the endcap calorimeter due to the increased flux and energy of the primary particles striking this region. Furthermore, the borated polyethylene reduces the flux by a factor of 2 to 5, depending on location. It reduces the flux both by decreasing the rate at which neutrons are injected in the central detector cavity, and by reducing the number of reflections. Increasing the borated polyethylene thickness does not always produce a proportionate reduction in the fluence. ${ }^{20}$ We believe that this is so because the polyethylene has a smaller effect on the high energy component of the albedo, and also 
because the polyethylene itself starts contributing to the albedo. The shielding configuration that we have chosen is shown in Figure 1b. We have designed the polyethylene in front of the endcap calorimeter to be thinner at larger angles so that it has as little effect on the overall electromagnetic resolution as possible. The average neutron flux in the central cavity is calculated to be $4 \times 10^{12} \mathrm{n} / \mathrm{cm}^{2} / S S C Y$; approximately half of these neutrons will be above $100 \mathrm{keV}$. The energy spectra for neutrons and photons are shown in Figure 14.

Now we consider the photon flux for the central detector cavity in more detail. Figure 13 shows the photon fluxes along the same surfaces for the different configurations of the polyethylene. There are two sources of photons in the central tracker: 1) albedo photons from electromagnetic showers and 2) photons from low energy neutron capture reactions in the material of the central detector cavity. The Monte Carlo calculation described in the previous paragraph simulates only photons from $(n, \gamma)$ reactions. The flux of electromagnetic albedo photons is approximately the same as the neutron flux ${ }^{10}$ without the borated polyethylene shield. The borated polyethylene shield reduces the neutron flux by an order of magnitude, but the additional photons resulting from neutron capture on boron increase the overall photon flux by a factor of two when added to the electromagnetic albedo. The average total photon flux in the center cavity with the borated polyethylene shield will be about $6 \times 10^{12}$ photons $/ \mathrm{cm}^{2} / S S C Y$.

The fluxes in the central tracker have the same uncertainties as the fluxes in the muon system discussed in the previous section. We have found that the GEANT-CALOR calculation gives neutron fluxes that are approximately a factor of 3 lower than the LAHET-MCNP calculation. The neutron spectrum in the central cavity from GEANT-CALOR is also somewhat softer. We have chosen to use the higher flux numbers from LAHET-MCNP in the interest of being conservative.

(a)

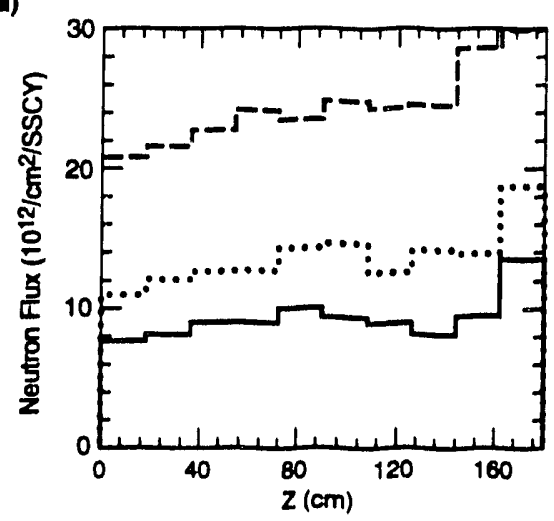

(c)

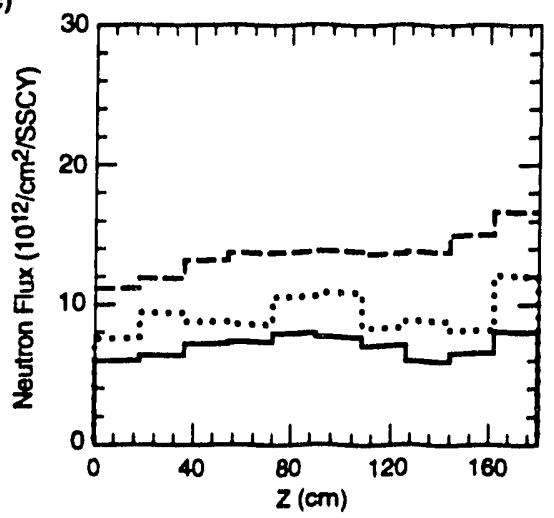

(b)

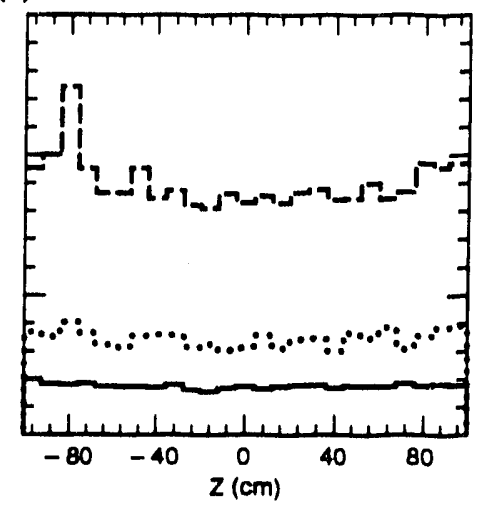

(d)

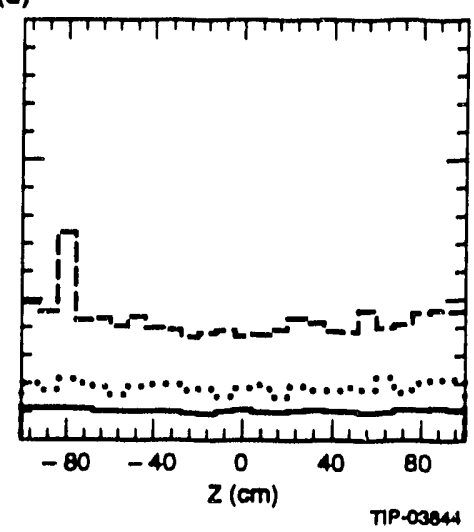

Figure 12. Flux of neutrons in units of $10^{12}$ neutrons $/ \mathrm{cm}^{2} / \mathrm{SSCY}$. a) Flux at a radius of $10 \mathrm{~cm}$ as a function of distance from the IP for all energles. b) Flux at a radlus of $44 \mathrm{~cm}$ as a function of distance from the IP for all energies. c) and d) are at the same radlus as a) and b) for neutrons with $E$ $>100 \mathrm{keV}$, respectlvely. The solid lines represent the borated polyethylene shielding shown in Figure 12-1b. The dotted lines indlcate $5 \mathrm{~cm}$-thlck borated polyethylene around the barrel and In front of the endcap. The dashed llnes represent no boratedpolyethylene shlelding. 
(a)

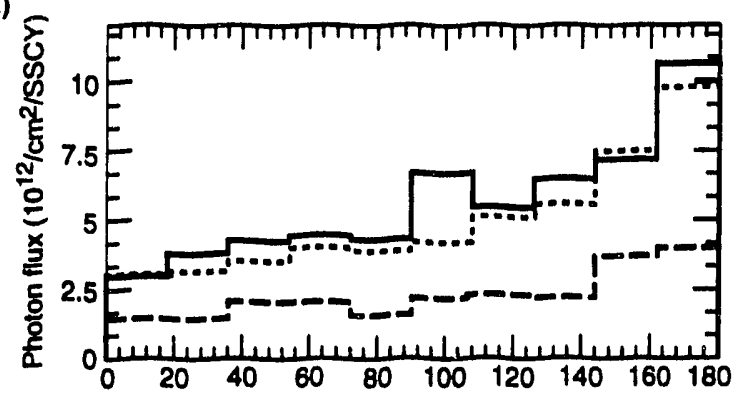

(b)

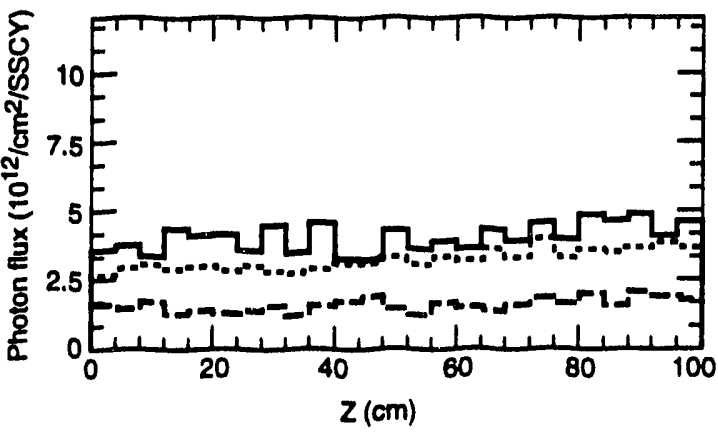

TP-03061

Figure 13. Flux of photons from $(n, \gamma)$ reactions in units of $10^{12}$ photons $/ \mathrm{cm}^{2} / \mathrm{SSCY}$ for two surfaces and for photons above $100 \mathrm{keV}$. a) Flux at a radlus of $10 \mathrm{~cm}$ as a function of distance from the IP. b) Flux at a radius of $44 \mathrm{~cm}$ as a function of distance from the IP. The solid lines represent the borated polyethylene shlelding shown In Fig. 12-1b. The dotted lines are for $5 \mathrm{~cm}$ thlck borated polyethylene around the barrel and In front of the endcap. The dashed lines are for no borated polyethylene shielding.

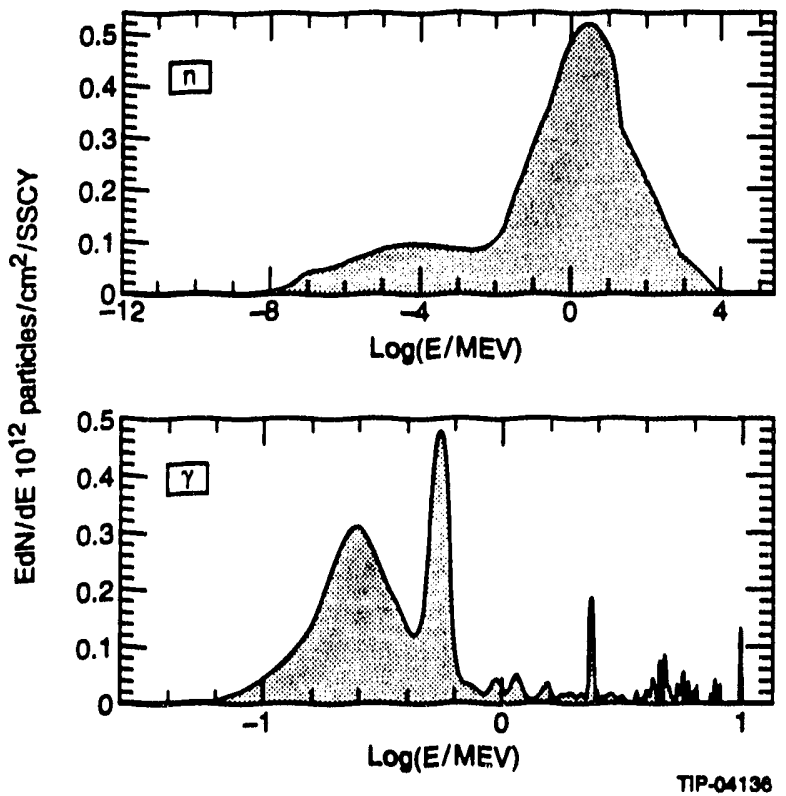

Figure 14. Neutron and photon energy spectra in the central tracker volume. These spectra were obtained at the innermost sillicon layer at a radlus of $10 \mathrm{~cm}$. The neutron spectrum includes high energy neutrons produced in the pp collisions. The photon spectrum only includes photons from ( $n, \gamma)$ reactlons. The peaks at $500 \mathrm{keV}$ and $2.2 \mathrm{MeV}$ are from captures on boron and hydrogen, respectively. The width of the peaks is mainly due to binning. 


\subsection{TOTAL RADIATION DOSE}

The detector components that will receive the highest radiation doses are located in the central detector cavity. These are: the silicon tracker and associated electronics, the IPC tracker and associated electronics, insulation of the cryostats for the electromagnetic calorimetry, the NEG vacuum pump, cables in the central cavity, and the beam pipe. From past experience at colliders, it is known that these systems can experience large instantaneous dose rates during unstable or unusual conditions in the accelerator. However, the parameters of the SSC are such that the majority of the integrated doses at interaction regions will result during normal operations. Therefore we have calculated the dose rates in different parts of the detector for standard SSC luminosity ( $10^{15}$ interactions/SSCY).

The radiation dose for each system can be divided into four categories: 1) dose due to ionization energy loss of charged particles from the interaction point, 2) dose due to electromagnetic showers initiated by electrons and photons from the interaction point, 3) dose due to hadronic showers initiated by hadrons from the interaction point, and 4) dose due to low energy neutrons resulting from of the hadronic cascades. In general, the first category, the $\mathrm{dE} / \mathrm{dx}$ loss, is dominant for thin objects such as the silicon detectors in the central tracker. Electromagnetic and hadronic showers contribute the majority of the dose to thick calorimeters. The maximum dose in calorimeters is dominated by electromagnetic showers simply because radiation lengths are much shorter than nuclear interaction lengths. We consider the dose due to low energy neutrons separately because the mechanism for energy deposition and damage-interactions on nuclei-is fundamentally different from ionization energy loss, which is the main mechanism in the first three cases.

\subsection{Charged Particle Dose}

We have calculated the $\mathrm{dE} / \mathrm{dx}$ dose rate to the silicon tracker due to charged particles from the interaction point by using the $\mathrm{dN} / \mathrm{d} \eta$ distribution. We have also taken into account secondary interactions, gamma conversions, and low energy charged particles, which spiral tightly in the magnetic field. We follow the calculation in Reference 10 with a few modifications relevant to the GEM tracker. The flux of charged particles at a radius $R$ from the beam line can be written as:

$$
\text { Charged Particle Flux }=\frac{\mathrm{K} L \sigma}{2 \pi R^{2}} \frac{\mathrm{dN}}{\mathrm{d} \eta} \text { particles } / \mathrm{cm}^{2}
$$

where $L$ is the integrated luminosity, $\sigma$ is the total $p p$ cross section, $\mathrm{dN} / \mathrm{d} \eta$ is the number of primary charged particles per unit of pseudorapidity per interaction, and $\mathrm{K}$ is the factor by which the flux is increased due to secondary interactions, loopers, and $\gamma$ conversions in the material of the tracker. According to Monte Carlo simulations of the GEM central tracker, $\mathrm{K}$ is estimated to be approximately 1.5. For one standard year of SSC running at $L=10^{33} \mathrm{~cm}^{-2} \mathrm{~s}^{-1}$ and a total $p p$ cross-section of $\sigma=10^{-25} \mathrm{~cm}^{2}$, with the expected value of $\mathrm{dN} / \mathrm{d} \eta \sim$ 7.5 charged particles/unit rapidity/event, we calculate at $R=10 \mathrm{~cm}$ from the beam line a flux of charged particles of $1.8 \times 10^{13}$ particles $/ \mathrm{cm}^{2} / \mathrm{SSCY}$. We can then write the radiation dose corresponding to a flux of charged particles as:

$$
\operatorname{Dose}(\mathrm{Mrad})=\frac{\text { charged particles } / \mathrm{cm}^{2}}{6.24 \times 10^{13}} \times \frac{\mathrm{dE}}{\mathrm{dx}}
$$

where $d E / d x$ is the energy loss of charged particles in a given material in units of $M e V /\left(\mathrm{gm} / \mathrm{cm}^{2}\right)$. We use $\mathrm{dE} / \mathrm{dx}=1.66 \mathrm{MeV} /\left(\mathrm{gm} / \mathrm{cm}^{2}\right)$ for silicon as a representative value for all momenta. The radiation dose for the silicon detector $10 \mathrm{~cm}$ away from the interaction point is $0.5 \mathrm{Mrad} / \mathrm{SSCY}$. At a tolerable lifetime dose of $5 \mathrm{Mrad}$ for silicon, this corresponds to a 10 year lifetime for the inner layer of silicon detectors at a radius of 10 $\mathrm{cm}$ and a luminosity of $10^{33} \mathrm{~cm}^{-2} \mathrm{~s}^{-1}$. In the vicinity of the IPC outer detector, at $40 \mathrm{~cm}$, the radiation dose is $30 \mathrm{krad} / \mathrm{SSCY}$. We are designing the IPC electronics to tolerate a lifetime dose of $3 \mathrm{Mrad}$, which corresponds to a lifetime of 10 years at a luminosity of $10^{34} \mathrm{~cm}^{-2} \mathrm{~s}^{-1}$. The dose rate in the tracker is plotted in Figure 15 . 


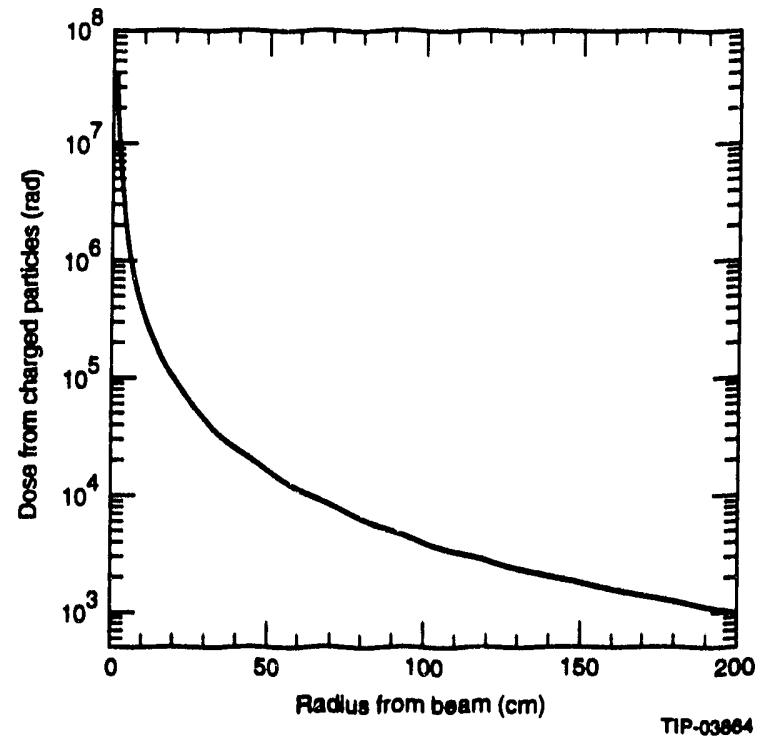

Figure 15. Dose to a silicon device as a function of radius in the central cavity due to lonizatiori energy loss of charged particles from the interaction point for one year of SSC operation at standard luminosity.

\subsection{Dose Due to Electromagnetic and Hadronic Showers}

We have used the parameterization of electromagnetic and hadronic showers from References $2: 3$ and 24 to compute the total dose in the calorimeter. The maximum dose occurs in the forward calorimeter. Figure 16 is a plot of the total dose in the forward calorimeter, including both electromagnetic and hadronic shower contributions for one year of operation at standard luminosity. This dose is plotted as a function of radius from the beam line and distance from the interaction point along the beam line. The maximum dose of $4.00 \mathrm{Mrad}$ at the inner comer of the urward hadronic calorimeter is dominated by electromagnetic showers initiated in the electromagnetic section. The hadronic dose is distributed over a much larger volume than the electromagnetic dose as expected from the ratio of interaction length to radiation length. The maximum hadroric dose of $10 \mathrm{Mrad}$ is deposited in the forward hadronic calorimeter. These numbers agree well with considerations in Reference 10.

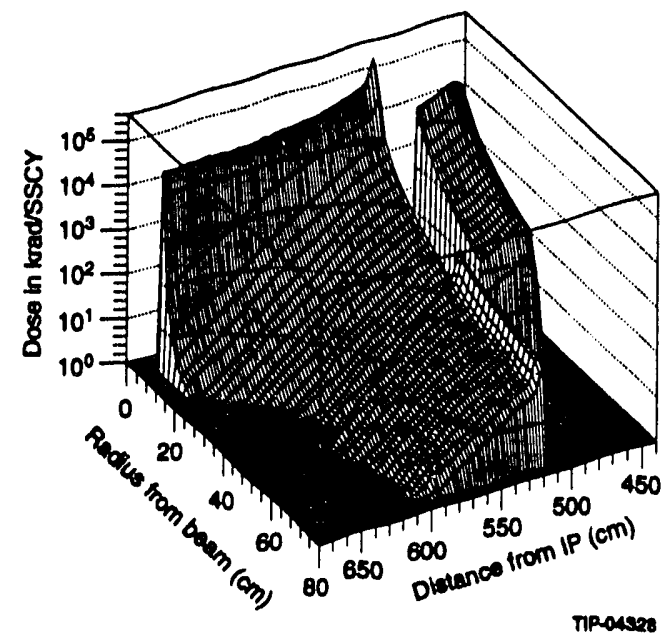

Flgure 16. Total dose due to electromagnetic and hadronic showers in the forward calorimeter for one year of SSC operation at standard luminosity. 


\subsection{Dose Due to Neutrons}

The damage from low energy neutrons can be divided in two parts: 1) damage due to ionization dose from emitted charged particles, recoiling nuclei, internal conversion, and radioactive decay; and 2) damage due to the displacement of atoms from their regular positions in a crystal lattice.

The ionization dose from neutron interactions can be estimated by folding the energy spectra of Figure 14 with tabulated heating factors (KERMA factors are another way of tabulating this information.) ${ }^{17,25}$ In particular, for the silicon in the central cavity we estimate a dose rate of approximately $1 \mathrm{krad} / \mathrm{SSCY}$, which is negligible compared to the ionization dose due to charged particles from the interaction point. Organic insulation on cables has a large heating factor because of the presence of hydrngen. We estimate a maximum dose rate of $0.1 \mathrm{Mrad} / \mathrm{SSCY}$ from neutrons behind the forward calorimeter for polyethylene insulation, corresponding to a lifetime of 100 years.

Damage to silicon due to displacement of atoms can be judged from existing data. ${ }^{21}$ If neutrons transfer a large amount of energy in a primary collision, the recoil Si itself continues to cause displacements. The result is a cluster of defects or a disordered region in the silicon crystal. To cause significant recoil damage, a neutron has to have an energy in excess of about $100 \mathrm{keV}$. It has been established that silicon detector performance degrades after exposure to more than $10^{14}$ neutrons $/ \mathrm{cm}^{2}$. Using this as the lifetime exposure "we obtain a lifetime greater than 10 years (from neutrons only) at standard luminosity for the inner most silicon layer in the central cavity.

We summarize the results of the study of total dose in Table 6. For design specifications of various detector components, we show the maximum doses from the different contributions for each detector system. For neutrons we list the maximum flux in the systems.

Table 6. Maximum dose and neutron flux expected in detector systems for one year of SSC operation at standard luminosity. The maximum neutron flux is as much as four times higher than the average.

\begin{tabular}{|c|c|c|c|c|}
\hline System & $\begin{array}{c}d E / d x \text { dose } \\
\text { (Mrad) }\end{array}$ & $\begin{array}{c}\text { EM and Had dose } \\
\text { (Mrad) }\end{array}$ & $\begin{array}{c}n \text { fluence } \\
\left(n / \mathrm{cm}^{2} / S S C Y \text { ) }\right.\end{array}$ & $\begin{array}{c}\text { n fluence for } \\
E 100 \mathrm{koV} \\
\left(\mathrm{n}^{\mathrm{cm}} / \mathrm{SSCY}\right)\end{array}$ \\
\hline Central tracker $\mathrm{Si}$ & 0.5 & - & $8 \times 10^{12}$ & $6 \times 10^{12}$ \\
\hline Central tracker IPC & 0.030 & - & $4 \times 10^{12}$ & $2 \times 10^{12}$ \\
\hline Barrel calorimeter & - & 0.1 & $5 \times 10^{12}$ & - \\
\hline Endcap calorimeter & - & 5.0 & $5 \times 10^{14}$ & - \\
\hline Forward EM calorimeter & - & 400 & $10^{15}$ & - \\
\hline Forward hadronic & - & 400 & $10^{15}$ & - \\
\hline Muon chambers & - & - & $6 \times 10^{10}$ & - \\
\hline
\end{tabular}

\subsection{ACTIVATION}

Residual activity levels in the detector must be considered both for personnel safety during access, and for computing the accidental hit rates in the sensitive detectors. In the following, we identify items in the detector that will be sufficiently activated to require special handling. We also show that the hit rates due to residual activity are negligibly low while data is being taken.

Radioactive nuclei in the detector are produced by several processes: spallation, nuclear breakup in high energy hadronic interactions and netitron scattering, and absorption of thermal neutrons. We expect the first two processes to dominate inside the calorimeters, because neutrons produced in hadronic showers in our calorimeters will be moderately energetic with small cross sections for absorption. Activation due to thermal 
neutron absorption will dominate in the muon system. We also note that the dose rates in the vicinity of thick extended objects are expected to be mainly from gamma radiation, while dose rates near thin objects such as the beam pipe and the muon chambers may have a significant contribution from beta emitting isotopes.

\subsection{CINDER Calculation of Activation}

We have performed a calculation of the activation of components in the GEM detector using the LAHET/MCNP code system in combination with the CINDER (Version C93A2) code. The LAHET code is used to predict the spectrum of residual nuclei from primary $p p$ interactions. In addition, neutron fluences under $20 \mathrm{MeV}$ computed by MCNP are used by CINDER to recreate the residual nuclei spectrum from low energy neutron interactions. The subsequent decay of these products is followed with the CINDER code. MCNP further transports the photons through the detector materials. The output of the entire program consists of a nuclide inventory, activity density, photon spectrum, and equivalent dose rates in rem/h at any given location in the detector as a function of time after irradiation. Table 7 shows the maximum expected dose rates, on contact, with no cooling time, in different detector systems after one year of SSC operation at standard luminosity. The calculation follows the decrease of dose rates for six months after the beam is turned off. Figure 17 shows the typical time dependence of the activity in the detector. Activity drops by a factor of 2 within a day after beam turn off at $10^{7} \mathrm{~s}$.

The above calculation was checked with the simple model of activation by Thomas and Stevenson, ${ }^{2}$ which converts the density of inelastic hadron interactions (stars) into equivalent dose rates. The dose rate in rem/h at saturation near a semi-infinite thick slab is given by $w \times\left(s t a r s / \mathrm{cm}^{3} / \mathrm{s}\right)$, where $w$ is a material dependent factor. For iron and most heavy metals $w=1.0 \times 10^{-6} \mathrm{~cm}^{3} \mathrm{~s} \mathrm{rem} / \mathrm{h}$. The last column in Table 7 shows the results of this calculation for some locations. Considering all the errors associated with these calculations the parameterization compares favorably with the CINDER calculation. Both sets of numbers have been scaled to be at the same location and time.

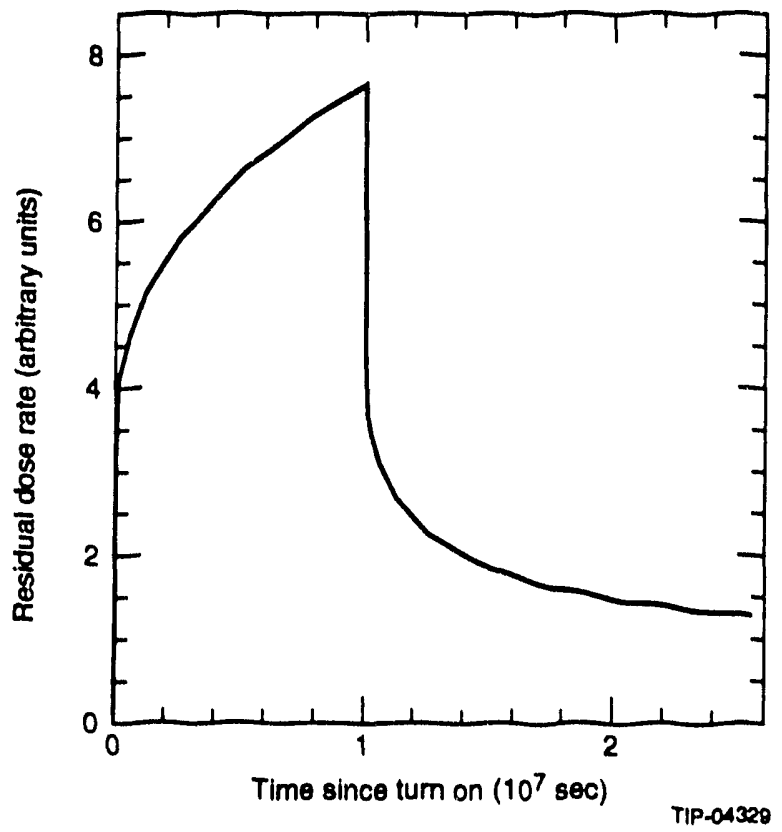

Figure 17. Bulldup and decay of residual dose rates in the GEM detector. The curve is normalized arbitrarlly. We assume an Irradiation time of $10^{7} 8$. 
Table 7. Maximum dose rates on contact for different regions of the detector from residual activity. The last column shows the results of a simple calculation based on the density of hadronic stars. The numbers are for one SSCY at standard luminosity. Activation in the muon system is mostly thermal neutron induced.

\begin{tabular}{|l|c|c|c|c|}
\hline SYSTEM & $\begin{array}{c}r \\
(\mathrm{~m})\end{array}$ & $\begin{array}{c}\mathbf{z} \\
(\mathrm{m})\end{array}$ & $\begin{array}{c}\text { CINDER } \\
(\mathrm{rem} / \mathrm{h})\end{array}$ & $\begin{array}{c}\text { STAR DENSITY } \\
(\mathrm{rem} / \mathrm{h})\end{array}$ \\
\hline Barrel & 1.0 & 0.0 & 0.002 & 0.01 \\
Endcap & 0.4 & 2.2 & 0.07 & 0.02 \\
EM forward & 0.06 & 4.5 & 4.5 & 1.1 \\
Hadronic forward & 0.10 & 6.5 & 0.50 & 2.0 \\
Field shaper & 0.60 & 10.0 & 0.10 & 0.02 \\
Pump & 0.10 & 9.0 & - & 0.04 \\
Collimator & 0.0 & 32.1 & 50.0 & 70.0 \\
\hline
\end{tabular}

\subsection{Activation Due to Low Energy Neutrons}

In this section, we consider the residual dose rates caused by thermal neutrons. The calculation using star density does not account for this contribution. The CINDER calculation does. The cross sections for activation by neutron absorption are largest for thermal neutrons; therefore, we pessimistically assume that half the neutrons are thermal everywhere outside and inside the calorimeter. In Table 8, we have reproduced a list of isotopes 12 with large cross sections for activation via thermal neutron absorption commonly present in high energy experiments. We have added some isotopes of krypton to this list, since we plan to use it in the barrel calorimeter. Using these cross sections and the thickness of copper in the muon chambers $(0.0034 \mathrm{~cm} /$ layer $)$, we estimate the occupancy in the muon chamber strips to be less than $10 \%$ of the total occupancy, which is small compared to occupancy levels expected from other sources. Similarly, we have estimated the pileup rate in the liquid krypton calorimeter due to krypton activation. We have found that the pileup noise level is less than $0.13 \mathrm{MeV} /$ tower, which is negligible compared to the thermal noise level/tower of $20 \mathrm{MeV}$.

Table 8. List of lsotopes with particularly high cross sections for activation via thermal neutron absorption. Most of the active lsotopes have several decay modes, including photons from short llved metastable states.

\begin{tabular}{|c|c|c|c|c|}
\hline Parent Isotope & Natural (\%) & $\sigma$ (Barns) & Active Isotope & Half Life \\
\hline${ }^{23} \mathrm{Na}$ & 100 & 0.53 & ${ }^{24} \mathrm{Na}$ & $15 \mathrm{~h}$ \\
${ }^{40} \mathrm{Ar}$ & 99.6 & 0.61 & ${ }^{41} \mathrm{Ar}$ & $1.8 \mathrm{~h}$ \\
${ }^{44} \mathrm{Ca}$ & 2.0 & 0.70 & ${ }^{45} \mathrm{Ca}$ & $165 \mathrm{~h}$ \\
${ }^{50} \mathrm{Cr}$ & 4.3 & 17 & $51 \mathrm{Cr}$ & $28 \mathrm{~d}$ \\
${ }^{55} \mathrm{Mn}$ & 100 & 13 & $66 \mathrm{Mn}$ & $2.58 \mathrm{~h}$ \\
${ }^{59} \mathrm{Co}$ & 100 & 37 & $60 \mathrm{Co}$ & $5.3 \mathrm{y}$ \\
${ }^{63} \mathrm{Cu}$ & 69 & 4.5 & $64 \mathrm{Cu}$ & $13 \mathrm{~h}$ \\
${ }^{64} \mathrm{Zn}$ & 49 & 0.46 & $65 \mathrm{Zn}$ & $245 \mathrm{~d}$ \\
$78 \mathrm{Kr}$ & 0.35 & 4.7 & $79 \mathrm{Kr}$ & $35 \mathrm{~h}$ \\
${ }^{80} \mathrm{Kr}$ & 2.27 & 4.6 & $61 \mathrm{Kr}$ & $13 \mathrm{~s}$ \\
${ }^{82} \mathrm{Kr}$ & 11.6 & 20 & $63 \mathrm{Kr}$ & $1.9 \mathrm{~h}$ \\
${ }^{84} \mathrm{Kr}$ & 57 & 0.10 & $65 \mathrm{Kr}$ & $10 \mathrm{y}$ \\
${ }^{12} \mathrm{Sb}$ & 57 & 6.1 & $122 \mathrm{Sb}$ & $2.8 \mathrm{~d}$ \\
$123 \mathrm{Sb}$ & 43 & 3.3 & $124 \mathrm{Sb}$ & $60 \mathrm{~d}$ \\
${ }^{133} \mathrm{Cs}$ & 100 & 31 & $134 \mathrm{Cs}$ & $2.1 \mathrm{y}$ \\
${ }^{151} \mathrm{Eu}$ & 48 & 8700 & $152 \mathrm{Eu}$ & $12 \mathrm{y}$ \\
${ }^{153} \mathrm{Eu}$ & 52 & 320 & $154 \mathrm{Eu}$ & $8 \mathrm{y}$ \\
${ }^{186} \mathrm{~W}$ & 28 & 40 & $187 \mathrm{~W}$ & $1 \mathrm{~d}$ \\
\hline
\end{tabular}




\subsection{SUMMARY}

We have designed a shielding configuration for the GEM detector that reduces the neutron fluxes in most of the muon system to the range of $1-5 \times 10^{10} \mathrm{n} / \mathrm{cm}^{2} / \mathrm{SSCY}$ at the standard luminosity of $10^{33} \mathrm{~cm}^{-2} \mathrm{~s}^{-1}$. The photon flux above $0.1 \mathrm{MeV}$ is approximately $30 \%$ of the neutron flux. The muon chambers surrounding the forward hadronic calorimeter are expected to receive neutron fluxes that are approximately twice as large. Because the GEM forward calorimeter is compact, and fits in the endcap calorimeter without leaving large gaps, it provides a natural, effective shield for the muon system. Our current results have been used to determine that the occupancy levels in the muon system are tolerable even at the high luminosity of $10^{34} \mathrm{~cm}^{-2} \mathrm{~s}^{-1}$.

We have also designed the shielding surrounding the central tracker, and estimated the neutron flux with this shielding to be about $4 \times 10^{12} \mathrm{n} / \mathrm{cm}^{2} / \mathrm{s}$ at standard luminosity. The photon flux is approximately twice as large above $0.1 \mathrm{MeV}$. This flux of neutrons and photons presents minimal rate difficulties for the silicon and IPC detectors in the central tracker. We expect the silicon tracker to perform without serious degradation for 10 years at standard luminosity $10^{33} \mathrm{~cm}^{-2} \mathrm{~s}^{-1}$; the limitation is caused mainly by the radiation dose in silicon due to charged particles.

We have quantified the total radiation doses to various systems in the detector. We have identified the forward calorimeter as the location of the maximum dose of about $400 \mathrm{Mrad}$ over one year of SSC operation at standard luminosity. We are designing this region with careful attention to the choice of materials with the expectation that the detector will eventually operate at 10 times higher luminosity.

We have quantified the residual activity and corresponding dose rates at various locations in the detector. In particular, we have identified forward regions, such as the forward calorimeter and the vacuum pump stations, that require special handling for radiation safety after one year of SSC operation.

This study required close co-ordination amongst many members of the GEM collaboration. We would especially like McNeil, Kate Morgan. 


\section{REFERENCES}

1. "GEM Technical Design Report," presented by the GEM collaboration, SSC-SR-1219, April 30, 1993.

2. J. Ranft, et al., in "Multiparticle Dynamics," Seewinkel, Austria, 1986. J. Ranft, Presentation in the Simulating Accelerator Radiation Environments Workshop, Santa Fe, New Mexico, Jan. 1993, to be published. See also J. Ranft, et al., UL-92-7 and UL-HEP-93-01, Leipzig, Germany. We have used the February 1, 1993 version of DTUJET.

3. N. Mokhov, "MARS12 code system," Presentation in the Simulating Accelerator Radiation Environments Workshop, Santa Fe, New Mexico, Jan. 1993, to be published. Also see N. Mokhov, MARS10 Code Manual, FERMILAB-FN-509, 1989.

4. "User Guide to LCS: The LAHET Code System," Richard E. Prael and Henry Lichtenstein, Los Alamos National Laboratory, LA-UR-89-3014, Sep. 1989. "MCNP: A General Monte Carlo Code for Neutron and Photon Transport," Judith F. Briesmeister, LA-7396-M Rev. 2, Sep. 1986. "Radiation Calculations using LAHET/MCNP/CINDER90," Proceedings of the III International Conference on Calorimetry in High Energy Physics, Corpus Christi, LA-UR-89-3014, Oct. 1992.

5. "CALOR: A Monte Carlo Program Package for the Design and Analysis of Calorimeter Systems," T. .A. Gabriel, J. D. Amburgey, B. L. Bishop, ORNL/TM-5619, Apr. 1977. "User's Guide for the FLUNEV Code," J. M.. Zazula, Desy-internal-rep D3-90-66, Jan. 1990. "FLUKA and KASPRO Hadronic Cascade Codes," J. Ranft, Erice 1978, Proceedings, Computer Techniques in Radiation Transport and Dosimetry.

6. "Hadronic Cascade Simulation in GEANT," K. Lassila, CERN-CN-91-13, Dec. 1991. "GEANT: Simulation Program for Particle Physics Experiments. Users Guide and Reference Manual," R. Brun, R. Hagelberg, M. Hansroul, J. C. Lassalle, CERN-DD/78/2 Rev. July 1978.

7. Y. Fisyak, K. McFarlane, L. Roberts, GEM-TN-92-162, 1992.

8. C. Zeitnitz, T. A. Gabriel, "The GEANT-CALOR Interface," III Conference for Calorimetry in High Energy Physics, Proceedings (to be published), Corpus Christi 1992. C. Zeitnitz, T. A. Gabriel, "The GEANT-CALOR Interface," International Conference on Monte Carlo Simulation in High Energy and Nuclear Physics, Proceedings (to be published), Tallahassee, 1993. See also J. O. Johnson, T. A. Gabriel, "Development and Evaluation of a Monte Carlo Code System for Analysis of Ionization Chamber Responses," ORNL/TM-10196, July 1987.

9. Description of CINDER90: Los Alamos Code for Estimation of Radio Activation," R. Wilson, Presentation in the Simulating Accelerator Radiation Environments Workshop, Santa Fe, New Mexico, Jan. 1993, to be published.

10. D. E. Groom, "Radiation Levels in the SSC Interaction Regions," SSC-SR-1033, June, 10, 1988.

11. Radiological Safety Aspects of the Operation of Proton Accelerators," R. H. Thomas and G. R. Stevenson, IAEA technical reports series no. 283, 1988.

12. A Guide to Radiation and Radioactivity Levels Near High Energy Particle Accelerators," A. H. Sullivan, Nuclear Technology Publishing, 1992. See also "Induced Radioactivity," Marcel Barbier, North Holland, 1969.

13. I. Baishev, A. Drozhdin, and N. Mokhov, SSC-306, 1991.

14. N. Mokhov, Collider ARC PDRR, May 26-27, 1992.

15. J. Ranft, private communication.

16. M. Diwan, "Design of a Compact Beam Dump for the BNL-AGS. Neutral Beam," SSCL-Preprint-192, Presentation in the Simulating Accelerator Radiation Environment Workshop, Santa Fe, New Mexico, Jan. 1993, to be published.

17. D. I. Garber, R. R. Kinsey, Neutron Cross Sections," BNL-325, Brookhaven National Laboratory, January 1976. See also "A Nuclear Cross Section Data Handbook," Harl'o M. Fisher, LA-11711-M-Manual, Los Alamos National Laboratory, Dec. 1989. 
18. Concrete Radiation Shielding: Nuclear Physics, Concrete Properties, Design and Construction," Wiley, New York, 1989. The concrete that we propose to use has a density of $3.2 \mathrm{gm} / \mathrm{cm}^{3}$; it contains, by weight, $40 \%$ barium, $35 \%$ oxygen, $9.6 \%$ sulfur, $8.5 \%$ calcium, $1.8 \%$ silicon, $1 \%$ iron, $1 \%$ boron, $0.85 \%$ hydrogen, and the rest are trace minerals.

19. "Neutron and Gamma Sensitivity of Gas Detectors," S. Boyarinov, et al., GEM-TN-93-345.

20. David M. Lee, R. E. Prael, Laurie Waters, GEM-TN-92-91, Apr. 1992.

21. "Radiation Resistance of Semiconductor Detectors and Associated Electronics," G. Hall, Presented at the LHC Workshop, Aachen, Oct. 1990, CERN 90-10, Vol. III, p. 693.

22. "Radiation Effects at the SSC," Edited by M. G. D. Gilchriese, SSCL-SR-1035, Jun. 1988. "Radiation Damage Testing at the SSC: Task Force Report," W. Chinowsky, R. Thun, SSC-SR-1054, Jun. 1990.

23. F. Barreiro, et al., "Measurements of Longitudinal and Transverse Profiles for Hadron Showers in the Range 10-100 GeV and Comparisons with Monte Carlo Simulations," NIM A292(1990) 259-278.

24. M. Della Negra, "Pattern Recognition in Calorimeters," Physica Scripta, Vol. 23, 469-479, 1981.

25. "Trends in Radiation Dosimetry," Caswell and Coyne, The Intemational Journal of Applied Radiation and Isotopes, Vol 33, No. 11, Nov. 1992.

26. G. R. Stevenson, CERN-TIS-RP-90-10-CF, August 1990. See also G. R. Stevenson, CERN-TIS-RP-90-18-CF, October 1990. 

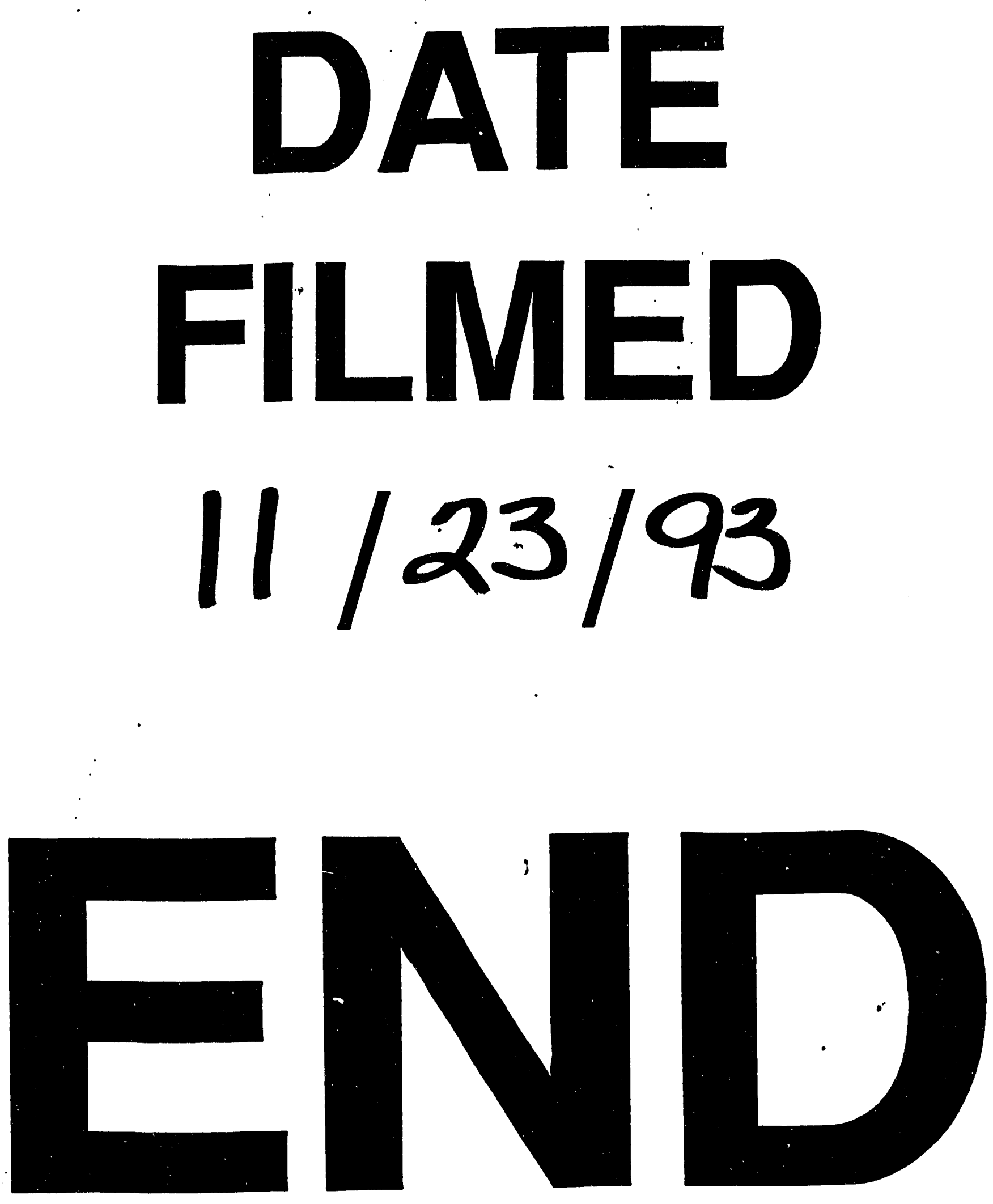
